\title{
Differences in the symptom experience of older versus younger oncology outpatients: a cross-sectional study
}

Janine K Cataldo ${ }^{1}$, Steven Paul ${ }^{1}$, Bruce Cooper ${ }^{1}$, Helen Skerman ${ }^{3}$, Kimberly Alexander ${ }^{3}$, Bradley Aouizerat ${ }^{1,4}$, Virginia Blackman ${ }^{1}$, John Merriman ${ }^{1}$, Laura Dunn², Christine Ritchie ${ }^{2}$, Patsy Yates ${ }^{3}$ and Christine Miaskowski ${ }^{1,5^{*}}$

\begin{abstract}
Background: Mortality rates for cancer are decreasing in patients under 60 and increasing in those over 60 years of age. The reasons for these differences in mortality rates remain poorly understood. One explanation may be that older patients received substandard treatment because of concerns about adverse effects. Given the paucity of research on the multiple dimensions of the symptom experience in older oncology patients, the purpose of this study was to evaluate for differences in ratings of symptom occurrence, severity, frequency, and distress between younger ( $<60$ years) and older ( $\geq 60$ years) adults undergoing cancer treatment. We hypothesized that older patients would have significantly lower ratings on four symptom dimensions.
\end{abstract}

Methods: Data from two studies in the United States and one study in Australia were combined to conduct this analysis. All three studies used the MSAS to evaluate the occurrence, severity, frequency, and distress of 32 symptoms.

Results: Data from 593 oncology outpatients receiving active treatment for their cancer (i.e., $44.4 \%$ were $<60$ years and $55.6 \%$ were $\geq 60$ years of age) were evaluated. Of the 32 MSAS symptoms, after controlling for significant covariates, older patients reported significantly lower occurrence rates for 15 (46.9\%) symptoms, lower severity ratings for 6 (18.9\%) symptoms, lower frequency ratings for 4 (12.5\%) symptoms, and lower distress ratings for 14 (43.8\%) symptoms.

Conclusions: This study is the first to evaluate for differences in multiple dimensions of symptom experience in older oncology patients. For almost 50\% of the MSAS symptoms, older patients reported significantly lower occurrence rates. While fewer age-related differences were found in ratings of symptom severity, frequency, and distress, a similar pattern was found across all three dimensions. Future research needs to focus on a detailed evaluation of patient and clinical characteristics (i.e., type and dose of treatment) that explain the differences in symptom experience identified in this study.

Keywords: Geriatric oncology, Symptoms

\footnotetext{
* Correspondence: chris.miaskowski@nursing.ucsf.edu

${ }^{1}$ School of Nursing, University of California, 2 Koret Way - N631Y, San

Francisco, CA 94143-0610, USA

${ }^{5}$ Department of Physiological Nursing, University of California, 2 Koret

Way - N631Y, San Francisco, CA 94143-0610, USA

Full list of author information is available at the end of the article
} 


\section{Background}

Cancer is predominantly an older person's illness. Approximately $50 \%$ of the most common cancers (i.e., breast, prostate, and lung) occur in people over 60 years of age [1]. Because of major advances in cancer treatment and supportive care strategies, a larger percentage of older persons are receiving active treatment [2]. However, while mortality rates for cancer are decreasing in patients under 60 , they are increasing in those over 60 years of age [1]. The reasons for these differences in mortality rates remain poorly understood primarily due to the paucity of research on older persons with cancer.

Older adults are a vulnerable population who are at high risk for suboptimal cancer care because they were under-represented in clinical trials and experience disparities in cancer treatment [3-7]. One reason why mortality rates may be increasing in patients over 60 is that they receive substandard treatment (i.e., lower doses of radiation therapy (RT) or chemotherapy (CTX)) because of the unsubstantiated belief that they will experience a larger number and more severe adverse effects as well as poor functional outcomes and significant decrements in quality of life (QOL) $[8,9]$. The foundations for these beliefs include the normal physiological changes that occur with aging that could influence drug metabolism, the co-occurrence of multiple co-morbidities in the elderly, and the increased risk of adverse effects associated with polypharmacy $[2,10,11]$. However, findings from studies on the impact of co-morbidities and cancer treatment on older people are contradictory [12-14].

In addition, in the limited number of studies that evaluated associations between age and the occurrence and severity of physical and psychological symptoms, the results are inconsistent. In a study of oncology patients undergoing RT [15], sleep disturbance, pain, and distress were significantly less prevalent among older patients compared to younger patients, while shortness of breath was significantly more prevalent among older patients before RT. In another study of newly diagnosed cancer patients [16], age was weakly correlated with ratings of symptom distress $(r=-0.11, \mathrm{p}<0.02)$ and older patients reported lower levels of symptom distress. In contrast, in a study of multiple symptoms [17], patients over 70 reported higher symptom distress scores than patients less than 40 years of age. In a study of patients with advanced cancer [10], compared to older patients, younger patients reported higher pain severity scores and better appetite. In terms of psychological symptoms, older age is consistently associated with lower levels of psychological distress regardless of clinical factors [18-21]. However, less is known about how older patients experience psychological distress associated with cancer and its treatment $[22,23]$.
In both Australia and the United States, the number of oncology patients over the age of 60 is growing exponentially $[1,24]$. In fact, estimates suggest that by 2030 , the incidence of cancer among older adults will increase by $67 \%$ [25]. If research studies are not done to characterize the symptom management needs of older persons with cancer, the medical systems in both countries will not be prepared to provide the supportive care interventions that these highly vulnerable patients need in order to receive appropriate treatments and survive their cancer. Therefore, given the paucity of research on age-related differences in the multiple dimensions of the symptom experience, the purpose of this study, using the Memorial Symptom Assessment Scale (MSAS) [26], was to evaluate for differences in ratings of symptom occurrence, severity, frequency, and distress between younger ( $<60$ years) and older ( $\geq 60$ years) adults undergoing cancer treatment. Based on previous studies [16,27-29], we hypothesized that older patients would have significantly lower ratings on all symptom dimensions than younger patients.

\section{Methods}

\section{Study samples}

Demographic, clinical, and symptom data from one Australian study (i.e., Symptom Clusters) and two United States studies (i.e., Fatigue, Pain, and Sleep Study (FPS study), Symptom Prevalence Study) were combined to conduct this analysis. All three studies enrolled patients who were receiving active treatment for their cancer. To evaluate the effect of age, patients were dichotomized into younger $(<60)$ and older $(\geq 60)$ groups based on cut-offs used in previous studies of cancer symptoms $[10,30,31]$, as well as findings that cancer mortality rates are increasing in those $>60$ years [1].

\section{Symptom clusters study}

This prospective, longitudinal study was designed to identify symptom clusters and their effects on physical and psychological functioning of patients with metastatic disease. Data were collected from patients using an interview-administered survey at the time of diagnosis or progression of metastatic disease and again at 2 months and 4 months. Data from the first assessment were used in these analyses.

Patients were recruited consecutively from two major tertiary referral hospitals in Australia: the Royal Brisbane and Women's Hospital and Peter MacCallum Cancer Centre (Melbourne). Patients were to participate if they: were adults ( $>18$ years of age) who could read, write, and understand English; had no cognitive limitations; had a primary cancer of breast, lung, colon/rectum, prostate, upper gastrointestinal tract, or ovaries; and were diagnosed with metastatic disease in the past 
month or had clinical evidence of progressive metastatic disease. Patients were excluded if they had local recurrence, but no evidence of metastatic disease; had a prognosis of $<4$ months as determined by their clinician; or had physical or cognitive impairments that precluded participation in the 15-minute survey. The study was approved by the Ethics Committees of Queensland University of Technology and the two participating hospitals.

Research staff liaised with clinical staff to identify potentially eligible patients, using a standard screening assessment sheet. All patients provided written consent prior to completing the study questionnaires. Of 306 patients approached, 218 patients were recruited $(71.2 \%$ response rate). Reasons for non-participation included: a clinician assessed that patients were not well enough to participate $(27.9 \%)$, prognosis $<4$ months $(22 \%)$, limited English (18.7\%), patient was currently participating in another study (18.4\%), and physically or cognitively unable to participate (13\%). The questionnaire was completed during a 20 minute face-to-face interview conducted by trained interviewers with nursing or psychology backgrounds. Clinical and demographic data were obtained from medical record reviews.

\section{FPS study}

This longitudinal study evaluated multiple symptoms in patients who underwent primary or adjuvant RT. Patients were recruited from two RT departments located in a Comprehensive Cancer Center and a community-based oncology program at the time of the patient's simulation visit. Patients were eligible to participate if they: were $\geq 18$ years of age; were scheduled to receive primary or adjuvant RT for one of four cancer diagnoses (i.e., breast, prostate, lung, brain); were able to read, write, and understand English; gave written informed consent; and had a Karnofsky Performance Status (KPS) score of $\geq 60$. Patients were excluded if they had: metastatic disease, more than one cancer diagnosis, or a diagnosed sleep disorder.

A total of 472 patients were approached and 185 consented to participate (response rate of $34 \%$ ). The primary reasons for refusal were being overwhelmed or too busy. The study was approved by the Committee on Human Research at the University of California, San Francisco (UCSF) and at the second site. At the time of the simulation visit (i.e., approximately one week prior to the initiation of RT), patients were approached by a research nurse to discuss participation in the study. After obtaining written informed consent, patients completed the study questionnaires. In addition, medical records were reviewed for disease and treatment information.

\section{Symptom prevalence study}

This descriptive, cross-sectional study used self-report questionnaires to obtain information from a convenience sample of oncology outpatients. Patients were recruited from four outpatient settings in Northern California, including a university-based Cancer Center, a Veterans Affairs facility, and two community-based outpatient clinics. Patients were eligible to participate if they were $>18$ years of age; were able to read, write, and understand English; gave written, informed consent; had KPS scores of $\geq 50$; and were receiving active cancer treatment.

A total of 310 patients were approached and 206 consented to participate (response rate of 66\%). The primary reasons for refusal were that a patient was too ill to participate $(80 \%)$, too busy (15\%), or not interested in the research study (5\%). Patients who agreed to participate provided written informed consent and were given a copy of the questionnaire booklet. They completed the study questionnaires in their home and returned them to the research office using a postage paid envelope. The study was approved by the Committee on Human Research at UCSF and at each of the study sites.

\section{Instruments}

\section{Demographic and clinical characteristics}

Demographic information on age, gender, marital status, and living arrangements were obtained at enrollment. Because of differences in the educational systems in Australia and the United States, data on education were recoded into a dichotomous variable (i.e., no post high school versus post high school education). In addition, patients' medical records were reviewed for cancer diagnosis, presence of metastatic disease, and current treatment regimens (i.e., none, CTX, RT, or both CTX and RT).

In the Australian study, patient's functional status was rated by their clinician using the Eastern Cooperative Oncology Group (ECOG) Performance Status score that ranges from 0 (fully active) to 4 (disabled) [32]. In the United States studies, patients rated their functional status using the KPS scale that ranged from 30 (I feel severely disabled and need to be hospitalized) to 100 (I feel normal; I have no complaints or symptoms). The KPS scale is widely used to evaluate functional status in patients with cancer and has well established validity and reliability. Based on the recommendations of Verger and colleagues [33], the KPS scores were converted to ECOG scores for use in subsequent analyses.

\section{Memorial Symptom Assessment Scale (MSAS)}

All three studies used the MSAS to evaluate the occurrence, severity, frequency, and distress of 32 symptoms commonly associated with cancer and its treatment [26]. The MSAS is a self-report questionnaire designed to measure the multidimensional experience of symptoms. Using the MSAS, patients were asked to indicate whether or not they had experienced each symptom in 
the past week (i.e., symptom occurrence). If they had experienced the symptom, they were asked to rate its frequency of occurrence, severity, and distress. Symptom frequency was evaluated using a 4-point Likert scale (i.e., 1 = rarely, 2 = occasionally, 3 = frequently, $4=$ almost constantly). Symptom severity was measured using a 4-point Likert scale (i.e., 1 = slight, 2 = moderate, $3=$ severe, $4=$ very severe). Symptom distress was measured using a 5-point Likert scale (i.e., $0=$ not at all, $1=$ a little bit, 2 = somewhat, 3 = quite a bit, $4=$ very much). Three subscale scores (i.e., physical, psychological, global distress index) and a total MSAS score were calculated. The reliability and validity of the MSAS is well established in studies of oncology inpatients and outpatients $[26,34]$. Cronbach's alphas for the total sample, as well as for patients $<60$ years and $\geq 60$ years are as follows: physical subscale $(.82, .82, .82)$, psychological subscale (.81, .80, .77), global distress index $(.82, .80, .83)$, and total MSAS score $(.88, .89, .87)$.

\section{Analysis}

The three data sets were combined and data were analyzed using SPSS version 19 and STATA SE Version 12. Descriptive statistics, means, and standard deviations for quantitative variables and frequencies and percentages for categorical variables were generated to describe various patient characteristics. Mean ratings of severity, frequency, and distress were calculated for those patients who reported the symptom. Because previous studies have dichotomized older and younger patients at age 60 $[10,30,31]$, older patients were defined as adults $\geq 60$ years of age. Independent sample t-tests and Chi Square analyses were used to evaluate for differences in demographic and clinical characteristics between the two age groups. Differences between the two age groups in characteristics with multiple levels (i.e., diagnosis, treatment, ECOG Performance Status score) were further examined using post-hoc contrasts with Bonferroni correction.

Logistic regression was used to evaluate the effect of increasing age on the occurrence rates of each symptom. Significant differences, between the two age groups ( $<60$ (younger) and $\geq 60$ (older) years of age), in the occurrence rates of each symptom were evaluated with binary logistic regression analyses. To examine the differences between the two age groups in the severity, frequency, and distress ratings of each symptom, ordinal logistic regression was utilized. Significant differences, between the two age groups $(<60$ (younger) and $\geq 60$ (older) years of age), in the MSAS subscale and total scores were evaluated with linear regression analyses. For all of the regression analyses, unadjusted and adjusted values (e.g., odds ratios and their corresponding 95\% confidence intervals) were generated for each symptom or scale score.

\section{Results}

Differences in demographic and clinical characteristics between older and younger patients

As shown in Table 1, data from 593 oncology outpatients (i.e., $44.4 \%$ were $<60$ years and $55.6 \%$ were $\geq 60$ years of age) were evaluated. No differences were found across the three studies, in the age distribution of the patients. In addition, no differences were found, across the three studies, in the mean age of the patients (i.e., Symptom Clusters Study $=62.2( \pm 12.0)$ years, FPS Study $=60.8 \quad( \pm 12.0) \quad$ years, Symptom Prevalence Study $=60.8( \pm 12.4)$ years; $\mathrm{F}(2,590)=.994, \mathrm{p}=.371)$. Across the three studies, the age distribution in 10 year increments was: 20 to $29=0.8 \%, 30$ to $39=4.2 \%, 40$ to $49=12.0 \%, 50$ to $59=27.3 \%, 60$ to $69=29.0 \%, 70$ to $79=21.4 \%$, and $\geq 80=5.3 \%$.

The mean age of the entire sample was $61.3( \pm 12.1)$ years. On average, younger patients were $50.3( \pm 7.7)$ and older patients were $70.1( \pm 6.5)$ years of age $(\mathrm{p}<.001)$. Older patients were: more likely to be male $(\mathrm{p}<.001)$; less likely to have finished high school; more likely to have prostate cancer $(\mathrm{p}<.001)$; more likely to be receiving RT $(\mathrm{p}<.001)$, and more likely to be fully active $(\mathrm{p}=.04)$.

\section{Differences in symptom occurrence rates between older and younger patients}

Table 2 provides the symptom occurrence rates for the total sample for each of the MSAS symptoms, as well as the unadjusted and adjusted odds of symptom occurrence as age increases by one year. After controlling for significant covariates (i.e., gender, education, diagnosis, treatment, and ECOG performance status), as age increased, significantly lower occurrence rates were reported for difficulty concentrating, pain, feeling nervous, nausea, difficulty sleeping, feeling bloated, vomiting, feeling sad, sweats, worrying, problems with sexual interest, feeling irritable, I don't look like myself, and changes in skin.

As shown in Table 3, after controlling for significant covariates, differences in occurrence rates between the two age groups were found for 15 (46.9\%) of the 32 MSAS symptoms. Older patients reported significantly lower occurrence rates for difficulty concentrating, pain, feeling nervous, nausea, feeling drowsy, difficulty sleeping, feeling bloated, vomiting, feeling sad, sweats, worrying, problems with sexual interest, feeling irritable, I don't look like myself, and changes in skin.

\section{Differences in symptom severity ratings between older and younger patients}

As shown in Table 4, after controlling for significant covariates, differences in severity scores were found for $6(18.9 \%)$ of the 32 MSAS symptoms. Older patients 
Table 1 Demographic and clinical characteristics for the total sample and differences between younger $(<60, n=263)$ and older $(\geq 60, n=330)$ patients

\begin{tabular}{|c|c|c|c|c|c|c|c|}
\hline \multirow[t]{2}{*}{ Characteristic } & \multirow[t]{2}{*}{ Total (\%) } & \multicolumn{2}{|c|}{ Age group } & \multirow{2}{*}{$\begin{array}{c}\mathbf{p}^{-} \\
\text {value }\end{array}$} & \multicolumn{3}{|c|}{ Post hoc contrasts } \\
\hline & & $<60(\%)$ & $\geq 60(\%)$ & & $<60(\%)$ & $\geq 60(\%)$ & $p$-value \\
\hline \multicolumn{8}{|l|}{ Study project } \\
\hline Fatigue, Pain, and Sleep & 28.8 & 28.9 & 28.8 & .170 & & & \\
\hline Symptom Prevalence & 34.4 & 38.0 & 31.5 & & & & \\
\hline Symptom Clusters & 36.8 & 33.1 & 39.7 & & & & \\
\hline Gender- Female & 54.6 & 70.7 & 41.8 & $<.001$ & & & \\
\hline Lives alone & 26.4 & 24.0 & 28.2 & .260 & & & \\
\hline Partnered/married & 60.9 & 57.3 & 63.7 & .126 & & & \\
\hline Education - Post high school & 61.4 & 68.8 & 55.5 & .001 & & & \\
\hline \multicolumn{8}{|l|}{ Diagnosis } \\
\hline Breast & 33.6 & 48.3 & 21.8 & & 48.3 & 21.8 & .0125 \\
\hline Prostate & 26.0 & 11.0 & 37.9 & $<.001$ & 11.0 & 37.9 & .0125 \\
\hline Lung & 13.2 & 11.4 & 14.5 & & & & \\
\hline Other & 27.3 & 29.3 & 25.8 & & & & \\
\hline Metastases & 34.9 & 37.3 & 32.9 & .297 & & & \\
\hline \multicolumn{8}{|l|}{ Treatment } \\
\hline None & 15.5 & 13.3 & 17.3 & & & & \\
\hline Only radiation & 43.6 & 33.8 & 51.4 & $<.001$ & 33.8 & 51.4 & .0125 \\
\hline Only chemotherapy & 27.4 & 31.2 & 24.3 & & & & \\
\hline Both & 13.5 & 21.7 & 7.0 & & 21.7 & 7.0 & .0125 \\
\hline \multicolumn{8}{|l|}{ ECOG Performance Status } \\
\hline Fully active & 21.4 & 15.8 & 26.0 & & 15.8 & 26.0 & .0100 \\
\hline Ambulatory, light work & 48.9 & 53.1 & 45.5 & .043 & & & \\
\hline Ambulatory, mobile $>50 \%$ & 21.6 & 23.5 & 20.1 & & & & \\
\hline Ambulatory, mobile $<50 \%$ & 7.2 & 6.5 & 7.7 & & & & \\
\hline Disabled & 0.9 & 1.2 & 0.6 & & & & \\
\hline Age (years; mean (standard deviation)) & $61.3(12.1)$ & $50.3(7.7)$ & $70.1(6.5)$ & $<.001$ & & & \\
\hline
\end{tabular}

Abbreviation: ECOG, Eastern Cooperative Oncology Group.

reported significantly lower severity scores for dry mouth, feeling drowsy, feeling sad, sweats, worrying, and I don't look like myself.

\section{Differences in symptom frequency ratings between older and younger patients}

As shown in Table 5, after controlling for significant covariates, differences in frequency scores were found for $4(12.5 \%)$ of the 32 MSAS symptoms. Older patients reported significantly lower frequency scores for feeling nervous, dry mouth, nausea, and feeling bloated.

\section{Differences in symptom distress ratings between older and younger patients}

As shown in Table 6, after controlling for significant covariates, differences in distress scores were found for 14 (43.8\%) of the 32 MSAS symptoms. Older patients reported significantly lower distress scores for pain, lack of energy, cough, dry mouth, nausea, feeling drowsy, difficulty sleeping, feeling bloated, feeling sad, sweats, worrying, hair loss, I don't look like myself, and changes in skin.

\section{Differences in MSAS subscale and total scores between younger and older patients}

As shown in Table 7, after controlling for significant covariates, older patients reported significantly lower scores on all of the MSAS subscales, as well as on the MSAS total score.

Differences in rankings of symptoms with highest occurrence, severity, frequency, and distress ratings between older and younger patients

Table 8 provides a summary of the differences in the rankings of the various symptom dimensions (i.e., occurrence, severity, frequency, distress) for the top 10 symptoms between older and younger patients. For symptom occurrence, the four most prevalent symptoms (i.e., lack 
Table 2 Symptom occurrence rates for the total sample and odds of symptom occurrence as age increases by one year

\begin{tabular}{|c|c|c|c|c|c|}
\hline \multirow[t]{2}{*}{ Symptom } & \multirow{2}{*}{$\begin{array}{l}\text { Occurrence } \\
\text { rates }(\%) \\
\text { for total } \\
\text { sample }\end{array}$} & \multicolumn{2}{|c|}{$\begin{array}{c}\text { Unadjusted values } \\
\text { Continuous age }\end{array}$} & \multicolumn{2}{|c|}{$\begin{array}{l}\text { Adjusted values } \\
\text { Continuous age }\end{array}$} \\
\hline & & Odds ratio & $\mathrm{Cl}$ & Odds ratio & $\mathrm{Cl}$ \\
\hline Difficulty concentrating & 45.9 & $.96+$ & $.95-.97$ & $.97+$ & $.96-.99$ \\
\hline Pain & 64.1 & $.97+$ & $.96-.98$ & $.97^{* *}$ & $.96-.99$ \\
\hline Lack of energy & 74.7 & $.98^{* *}$ & $.96-.99$ & .99 & $.97-1.01$ \\
\hline Cough & 37.6 & 1.00 & $.98-1.01$ & 1.00 & $.98-1.02$ \\
\hline Feeling nervous & 32.4 & $.98^{* *}$ & $.96-.99$ & $.98^{*}$ & $.97-.99$ \\
\hline Dry mouth & 42.8 & 1.00 & $.99-1.01$ & 1.01 & $.99-1.03$ \\
\hline Nausea & 31.2 & $.98^{* *}$ & $.97-.99$ & $.98^{*}$ & $.97-.99$ \\
\hline Feeling drowsy & 58.7 & .99 & $.97-1.00$ & .99 & $.98-1.01$ \\
\hline Numbness/tingling in hands/feet & 31.9 & .99 & $.97-1.00$ & .99 & $.98-1.01$ \\
\hline Difficulty sleeping & 53.3 & $.96+$ & $.95-.98$ & $.97^{* *}$ & $.96-.99$ \\
\hline Feeling bloated & 24.8 & $.97+$ & $.95-.98$ & $.97+$ & $.95-.98$ \\
\hline Problems with urination & 29.5 & $1.03+$ & $1.02-1.05$ & 1.01 & $.99-1.03$ \\
\hline Vomiting & 13.8 & $.98^{*}$ & $.97-.99$ & $.97^{*}$ & $.95-.99$ \\
\hline Shortness of breath & 34.6 & .99 & $.98-1.00$ & .99 & $.97-1.00$ \\
\hline Diarrhea & 21.8 & 1.00 & $.99-1.02$ & 1.00 & $.98-1.02$ \\
\hline Feeling sad & 40.5 & $.95+$ & $.94-.97$ & $.96+$ & $.94-.97$ \\
\hline Sweats & 37.4 & $.98^{* *}$ & $.96-.99$ & $.98^{*}$ & $.96-.99$ \\
\hline Worrying & 43.5 & $.95+$ & $.94-.96$ & $.95+$ & $.94-.97$ \\
\hline Problems with sexual interest & 28.3 & $.97+$ & $.95-.98$ & $.95+$ & $.93-.97$ \\
\hline Itching & 28.0 & $.98^{*}$ & $.97-.99$ & .99 & $.98-1.01$ \\
\hline Lack of appetite & 33.9 & .99 & $.97-1.00$ & .99 & $.98-1.01$ \\
\hline Dizziness & 23.8 & .99 & $.98-1.01$ & .99 & $.98-1.01$ \\
\hline Difficulty swallowing & 15.7 & 1.00 & $.98-1.02$ & 1.00 & $.98-1.02$ \\
\hline Feeling irritable & 40.8 & $.96+$ & $.95-.97$ & $.96+$ & $.95-.98$ \\
\hline Mouth sores & 13.7 & 1.00 & $.98-1.02$ & 1.01 & $.99-1.03$ \\
\hline Changes in the way food tastes & 30.5 & 1.00 & $.99-1.02$ & 1.01 & $.99-1.03$ \\
\hline Weight loss & 23.1 & .99 & $.98-1.01$ & 1.00 & $.98-1.02$ \\
\hline Hair loss & 20.7 & $.98^{*}$ & $.97-.99$ & .99 & $.97-1.01$ \\
\hline Constipation & 32.2 & 1.00 & $.99-1.01$ & 1.00 & $.98-1.01$ \\
\hline Swelling of arms or legs & 16.4 & 1.01 & $.99-1.03$ & 1.02 & $1.00-1.04$ \\
\hline I don't look like myself & 27.3 & $.97+$ & $.95-.98$ & $.98^{*}$ & $.96-.99$ \\
\hline Changes in skin & 28.2 & $.96+$ & $.94-.97$ & $.98^{* *}$ & $.96-.99$ \\
\hline
\end{tabular}

Abbreviation: $\mathrm{Cl}$, confidence interval.

${ }^{*} \mathrm{p}<.05,{ }^{* *} \mathrm{p}<.01,+\mathrm{p}<.001$.

of energy, pain, feeling drowsy, difficulty sleeping) were the same for both age groups. Eight of 10 symptoms $(80 \%)$ were in the top 10 for both age groups. Feeling sad and sweats were unique to younger patients. Problems with urination, cough, and shortness of breath were unique to older patients.

For symptom severity, the four most severe symptoms (i.e., problems with sexual interest, hair loss, constipation, difficulty sleeping) were the same for both age groups. Seven of 10 symptoms (70\%) were in the top 10 for both age groups. Sweats, I don't look like myself, and worrying were unique to younger patients. Problems with urination, shortness of breath, and lack of appetite were unique to older patients.

For symptom frequency, the three most frequent symptoms (i.e., problems with sexual interest, hair loss, lack of energy) were the same for both age groups. Seven of 10 symptoms $(70 \%)$ were in the top 10 for both age groups. Dry mouth, I don't look like myself, and changes in skin were unique to younger patients. Problems with urination, lack of appetite, and changes in taste were unique to older patients.

For symptom distress, two symptoms (i.e., problems with sexual interest, constipation) were ranked as the 
Table 3 Differences in symptom OCCURRENCE rates between younger $(<60, n=263)$ and older $(\geq 60, n=330)$ patients

\begin{tabular}{|c|c|c|c|c|c|c|}
\hline \multirow[t]{2}{*}{ Symptom } & \multicolumn{2}{|c|}{$\begin{array}{l}\text { Occurrence rates } \\
\text { (\%) by age group }\end{array}$} & \multicolumn{2}{|c|}{$\begin{array}{c}\text { Unadjusted values } \\
\text { Older age }\end{array}$} & \multicolumn{2}{|c|}{$\begin{array}{l}\text { Adjusted values } \\
\text { Older age }\end{array}$} \\
\hline & $<60$ & $\geq 60$ & Odds ratio & $\mathrm{Cl}$ & Odds ratio & $\mathrm{Cl}$ \\
\hline Difficulty concentrating & 57.0 & 37.0 & $.44+$ & $.32-.62$ & $.58^{* *}$ & $.40-.85$ \\
\hline Pain & 73.0 & 57.0 & $.49+$ & $.35-.69$ & $.51^{* *}$ & $.34-.75$ \\
\hline Lack of energy & 81.7 & 69.1 & $.50+$ & $.34-.74$ & .69 & $.44-1.08$ \\
\hline Cough & 40.3 & 35.5 & .81 & $.58-1.14$ & .89 & $.60-1.32$ \\
\hline Feeling nervous & 39.5 & 26.7 & $.56^{* *}$ & $.39-.79$ & $.61^{*}$ & $.41-.91$ \\
\hline Dry mouth & 43.7 & 42.1 & .94 & $.68-1.30$ & 1.10 & $.75-1.62$ \\
\hline Nausea & 38.0 & 25.8 & $.57^{* *}$ & $.40-.80$ & $.52^{* *}$ & $.34-.79$ \\
\hline Feeling drowsy & 65.0 & 53.6 & $.62^{* *}$ & $.45-.87$ & $.68^{*}$ & $.47-.99$ \\
\hline Numbness/tingling in hands/feet & 36.1 & 28.5 & $.70^{*}$ & $.50-.99$ & .76 & $.51-1.14$ \\
\hline Difficulty sleeping & 63.9 & 44.8 & $.46+$ & $.33-.64$ & $.55^{* *}$ & $.38-.80$ \\
\hline Feeling bloated & 31.9 & 19.1 & $.50+$ & $.35-.73$ & $.51^{* *}$ & $.33-.78$ \\
\hline Problems with urination & 21.3 & 36.1 & $2.09+$ & $1.44-3.02$ & 1.18 & $.76-1.83$ \\
\hline Vomiting & 17.5 & 10.9 & $.58^{*}$ & $.36-.92$ & $.41^{* *}$ & $.23-.72$ \\
\hline Shortness of breath & 37.6 & 32.1 & .78 & $.56-1.10$ & .73 & $.48-1.11$ \\
\hline Diarrhea & 21.7 & 21.8 & 1.01 & $.68-1.49$ & .86 & $.54-1.35$ \\
\hline Feeling sad & 52.1 & 31.2 & $.42+$ & $.30-.58$ & $.46+$ & $.31-.67$ \\
\hline Sweats & 45.2 & 31.2 & $.55+$ & $.39-.77$ & $.60^{* *}$ & $.41-.88$ \\
\hline Worrying & 57.8 & 32.1 & $.35+$ & $.25-.48$ & $.39+$ & $.27-.56$ \\
\hline Problems with sexual interest & 35.7 & 22.4 & $.52+$ & $.36-.75$ & $.34+$ & $.22-54$ \\
\hline Itching & 34.2 & 23.0 & $.58^{* *}$ & $.40-.83$ & .72 & $.47-1.08$ \\
\hline Lack of appetite & 38.0 & 30.6 & .72 & $.51-1.01$ & .68 & $.45-1.02$ \\
\hline Dizziness & 27.0 & 21.2 & .73 & $.50-1.06$ & .71 & $.46-1.10$ \\
\hline Difficulty swallowing & 16.3 & 15.2 & .91 & $.59-1.43$ & .92 & $.55-1.56$ \\
\hline Feeling irritable & 51.7 & 32.1 & $.44+$ & $.32-.62$ & $.47+$ & $.32-.69$ \\
\hline Mouth sores & 14.8 & 12.7 & .84 & $.52-1.34$ & .91 & $.54-1.54$ \\
\hline Changes in the way food tastes & 31.2 & 30.0 & .95 & $.67-1.34$ & 1.09 & $.71-1.68$ \\
\hline Weight loss & 23.2 & 23.0 & .99 & $.68-1.46$ & 1.10 & $.69-1.73$ \\
\hline Hair loss & 23.6 & 18.5 & .74 & $.49-1.09$ & .90 & $.55-1.49$ \\
\hline Constipation & 33.8 & 30.9 & .87 & $.62-1.24$ & .82 & $.55-1.22$ \\
\hline Swelling of arms or legs & 16.3 & 16.4 & 1.00 & $.65-1.55$ & 1.10 & $.66-1.82$ \\
\hline I don't look like myself & 35.4 & 20.9 & $.48+$ & $.34-.70$ & $.64^{*}$ & $.42-.98$ \\
\hline Changes in skin & 38.0 & 20.3 & $.42+$ & $.29-60$ & $.62^{*}$ & $.41-.94$ \\
\hline
\end{tabular}

Abbreviation: $\mathrm{Cl}$, confidence interval.

${ }^{*} \mathrm{p}<.05,{ }^{* *} \mathrm{p}<.01,+\mathrm{p}<.001$.

first or second most distressing symptom in the two age groups. Six of 10 symptoms (60\%) were in the top 10 for both age groups. Nausea, feeling bloated, feeling sad, and I don't look like myself were unique to younger patients. Problems with urination, shortness of breath, difficulty swallowing, and swelling of arms or legs were unique to older patients.

\section{Discussion}

This study is the first to evaluate for differences in multiple dimensions of the symptom experience (i.e., occurrence, severity, frequency, and distress) between older and younger adults undergoing active treatment for cancer. The majority of the age differences were found in ratings of symptom occurrence with older patients reporting significantly lower occurrence rates for 15 (46.9\%) of the MSAS symptoms. While fewer age-related differences were found in ratings of symptom severity, frequency, and distress, for younger and older patients who reported a specific symptom, a similar pattern was found across all three dimensions, namely when differences occurred older patients reported significantly lower ratings.

While this secondary analysis provides interesting and important data on age-related differences in various 
Table 4 Differences in SEVERITY ratings between younger $(<60, n=263)$ and older $(\geq 60, n=330)$ patients

\begin{tabular}{|c|c|c|c|c|c|c|c|c|c|c|c|c|c|}
\hline \multirow[t]{2}{*}{ Symptom } & \multirow[t]{2}{*}{$\begin{array}{l}\text { Sample } \\
\text { size }\end{array}$} & \multirow[t]{2}{*}{$\begin{array}{l}\text { Age } \\
\text { group }\end{array}$} & \multirow[t]{2}{*}{$\begin{array}{l}\text { Mean } \\
\text { score }\end{array}$} & \multicolumn{4}{|c|}{ Severity ratings $(\%)$} & \multicolumn{3}{|c|}{$\begin{array}{l}\text { Unadjusted values } \\
\text { Older age }\end{array}$} & \multicolumn{3}{|c|}{$\begin{array}{l}\text { Adjusted values } \\
\text { Older age }\end{array}$} \\
\hline & & & & 1 & 2 & 3 & 4 & OR & $\mathrm{Cl}$ & p-value & OR & $\mathrm{Cl}$ & p-value \\
\hline \multirow[t]{2}{*}{ Difficulty concentrating } & 149 & $<60$ & 1.67 & 44.3 & 45.0 & 10.1 & 0.7 & .80 & $.50-1.28$ & .353 & .78 & $.46-1.32$ & .354 \\
\hline & 115 & $\geq 60$ & 1.61 & 50.4 & 40.0 & 7.8 & 1.7 & & & & & & \\
\hline \multirow[t]{2}{*}{ Pain } & 192 & $<60$ & 2.08 & 24.5 & 47.9 & 22.9 & 4.7 & 1.08 & $.74-1.58$ & .674 & .97 & $.64-1.49$ & .898 \\
\hline & 181 & $\geq 60$ & 2.12 & 24.3 & 45.3 & 24.3 & 6.1 & & & & & & \\
\hline \multirow[t]{2}{*}{ Lack of energy } & 214 & $<60$ & 2.15 & 15.4 & 57.5 & 23.8 & 3.3 & .91 & $.64-1.31$ & .622 & .81 & $.55-1.21$ & .311 \\
\hline & 217 & $\geq 60$ & 2.13 & 21.7 & 49.8 & 22.1 & 6.5 & & & & & & \\
\hline \multirow[t]{2}{*}{ Cough } & 102 & $<60$ & 1.74 & 46.1 & 36.3 & 15.7 & 2.0 & 1.04 & $.63-1.73$ & .870 & .86 & $.47-1.55$ & .609 \\
\hline & 110 & $\geq 60$ & 1.75 & 44.5 & 38.2 & 14.5 & 2.7 & & & & & & \\
\hline \multirow[t]{2}{*}{ Feeling nervous } & 103 & $<60$ & 1.83 & 35.9 & 47.6 & 13.6 & 2.9 & .81 & $.47-1.40$ & .452 & .81 & $.42-1.54$ & .514 \\
\hline & 85 & $\geq 60$ & 1.78 & 40.0 & 48.2 & 5.9 & 5.9 & & & & & & \\
\hline \multirow[t]{2}{*}{ Dry mouth } & 110 & $<60$ & 2.06 & 28.2 & 41.8 & 25.5 & 4.5 & .64 & $.40-1.03$ & .068 & .54 & $.32-.91$ & .020 \\
\hline & 131 & $\geq 60$ & 1.89 & 38.2 & 41.2 & 14.5 & 6.1 & & & & & & \\
\hline \multirow[t]{2}{*}{ Nausea } & 99 & $<60$ & 1.99 & 31.3 & 42.4 & 22.2 & 4.0 & .64 & $.37-1.10$ & .107 & 62 & $.33-1.14$ & .123 \\
\hline & 81 & $\geq 60$ & 1.80 & 43.2 & 37.0 & 16.0 & 3.7 & & & & & & \\
\hline \multirow[t]{2}{*}{ Feeling drowsy } & 167 & $<60$ & 1.95 & 24.6 & 58.1 & 15.6 & 1.8 & .68 & $.45-1.03$ & .068 & .61 & $.38-.96$ & .033 \\
\hline & 169 & $\geq 60$ & 1.82 & 32.5 & 55.0 & 10.7 & 1.8 & & & & & & \\
\hline \multirow[t]{2}{*}{ Numbness/tingling in hands/feet } & 94 & $<60$ & 1.85 & 36.2 & 45.7 & 14.9 & 3.2 & .90 & $.53-1.55$ & .710 & .79 & $.42-1.52$ & .484 \\
\hline & 91 & $\geq 60$ & 1.85 & 41.8 & 38.5 & 13.2 & 6.6 & & & & & & \\
\hline \multirow[t]{2}{*}{ Difficulty sleeping } & 168 & $<60$ & 2.23 & 14.9 & 54.8 & 23.2 & 7.1 & .87 & $.56-1.33$ & .512 & .67 & $.42-1.09$ & .108 \\
\hline & 141 & $\geq 60$ & 2.16 & 17.0 & 54.6 & 24.1 & 4.3 & & & & & & \\
\hline \multirow[t]{2}{*}{ Feeling bloated } & 82 & $<60$ & 1.91 & 30.5 & 51.2 & 14.6 & 3.7 & .77 & $.40-1.47$ & .429 & .77 & $.36-1.66$ & .510 \\
\hline & 55 & $\geq 60$ & 1.82 & 36.4 & 49.1 & 10.9 & 3.6 & & & & & & \\
\hline \multirow[t]{2}{*}{ Problems with urination } & 54 & $<60$ & 1.96 & 33.3 & 42.6 & 18.5 & 5.6 & 1.14 & $.61-2.11$ & .685 & 1.10 & $.56-2.18$ & .777 \\
\hline & 110 & $\geq 60$ & 2.01 & 26.4 & 52.7 & 14.5 & 6.4 & & & & & & \\
\hline Vomiting & 43 & $<60$ & 1.91 & 41.9 & 32.6 & 18.6 & 7.0 & .90 & $.38-2.14$ & .811 & .51 & $.17-1.54$ & .232 \\
\hline & 29 & $\geq 60$ & 1.79 & 37.9 & 44.8 & 17.2 & 0.0 & & & & & & \\
\hline Shortness of breath & 97 & $<60$ & 1.78 & 40.2 & 45.4 & 10.3 & 4.1 & 1.52 & $.90-2.58$ & .116 & 1.27 & $.71-2.29$ & .423 \\
\hline & 99 & $\geq 60$ & 2.00 & 33.3 & 42.4 & 15.2 & 9.1 & & & & & & \\
\hline Diarrhea & 54 & $<60$ & 1.93 & 24.1 & 61.1 & 13.0 & 1.9 & .67 & $.33-1.35$ & .263 & .62 & $.27-1.42$ & .258 \\
\hline & 63 & $\geq 60$ & 1.81 & 36.5 & 49.2 & 11.1 & 3.2 & & & & & & \\
\hline Feeling sad & 137 & $<60$ & 1.99 & 30.7 & 43.8 & 21.2 & 4.4 & .43 & $.26-.72$ & .001 & .43 & $.24-.76$ & .003 \\
\hline & 93 & $\geq 60$ & 1.66 & 46.2 & 46.2 & 3.2 & 4.3 & & & & & & \\
\hline Sweats & 117 & $<60$ & 2.14 & 23.9 & 44.4 & 25.6 & 6.0 & .54 & $.33-.91$ & .019 & .56 & $.31-.997$ & .049 \\
\hline & 95 & $\geq 60$ & 1.86 & 34.7 & 46.3 & 16.8 & 2.1 & & & & & & \\
\hline Worrying & 152 & $<60$ & 2.10 & 20.4 & 58.6 & 11.8 & 9.2 & .43 & $.26-.72$ & .001 & .52 & $.30-.90$ & .020 \\
\hline & 101 & $\geq 60$ & 1.77 & 38.6 & 49.5 & 7.9 & 4.0 & & & & & & \\
\hline Problems with sexual interest & 92 & $<60$ & 2.50 & 22.8 & 31.5 & 18.5 & 27.2 & 1.43 & $.82-2.52$ & .212 & 1.12 & $.57-2.19$ & .745 \\
\hline & 69 & $\geq 60$ & 2.72 & 18.8 & 24.6 & 21.7 & 34.8 & & & & & & \\
\hline Itching & 88 & $<60$ & 1.86 & 38.6 & 43.2 & 11.4 & 6.8 & .84 & $.46-1.52$ & .562 & .89 & $.46-1.75$ & .745 \\
\hline & 69 & $\geq 60$ & 1.78 & 42.0 & 43.5 & 8.7 & 5.8 & & & & & & \\
\hline Lack of appetite & 99 & $<60$ & 2.00 & 27.3 & 49.5 & 19.2 & 4.0 & .96 & $.57-1.64$ & .886 & .77 & $.42-1.43$ & .409 \\
\hline & 94 & $\geq 60$ & 2.00 & 27.7 & 51.1 & 14.9 & 6.4 & & & & & & \\
\hline Dizziness & 69 & $<60$ & 1.54 & 55.1 & 37.7 & 5.8 & 1.4 & .84 & $.43-1.65$ & .615 & .61 & $.27-1.39$ & .241 \\
\hline & 64 & $\geq 60$ & 1.48 & 59.4 & 34.4 & 4.7 & 1.6 & & & & & & \\
\hline Difficulty swallowing & 40 & $<60$ & 2.03 & 27.5 & 45.0 & 25.0 & 2.5 & .53 & $.23-1.21$ & .133 & .39 & $.14-1.10$ & .074 \\
\hline
\end{tabular}


Table 4 Differences in SEVERITY ratings between younger $(<60, \mathbf{n}=\mathbf{2 6 3})$ and older $(\geq 60, \mathbf{n}=\mathbf{3 3 0})$ patients (Continued)

\begin{tabular}{|c|c|c|c|c|c|c|c|c|c|c|c|c|c|}
\hline & 43 & $\geq 60$ & 1.79 & 37.2 & 51.2 & 7.0 & 4.7 & & & & & & \\
\hline \multirow[t]{2}{*}{ Feeling irritable } & 135 & $<60$ & 1.77 & 40.7 & 42.2 & 16.3 & 0.7 & .82 & $.50-1.34$ & .425 & .65 & $.37-1.15$ & .142 \\
\hline & 98 & $\geq 60$ & 1.68 & 41.8 & 49.0 & 8.2 & 1.0 & & & & & & \\
\hline \multirow[t]{2}{*}{ Mouth sores } & 37 & $<60$ & 1.86 & 43.2 & 35.1 & 13.5 & 8.1 & .55 & $.23-1.34$ & .188 & .52 & $.19-1.43$ & .205 \\
\hline & 35 & $\geq 60$ & 1.60 & 60.0 & 22.9 & 14.3 & 2.9 & & & & & & \\
\hline \multirow[t]{2}{*}{ Change in way food tastes } & 79 & $<60$ & 2.06 & 27.8 & 48.1 & 13.9 & 10.1 & .81 & $.46-1.43$ & .476 & 1.05 & $.57-1.93$ & .883 \\
\hline & 91 & $\geq 60$ & 1.96 & 31.9 & 47.3 & 14.3 & 6.6 & & & & & & \\
\hline \multirow[t]{2}{*}{ Weight loss } & 60 & $<60$ & 1.67 & 48.3 & 38.3 & 11.7 & 1.7 & .87 & $.45-1.69$ & .674 & .60 & $.28-1.30$ & .196 \\
\hline & 67 & $\geq 60$ & 1.63 & 52.2 & 35.8 & 9.0 & 3.0 & & & & & & \\
\hline \multirow[t]{2}{*}{ Hair loss } & 61 & $<60$ & 2.38 & 27.9 & 26.2 & 26.2 & 19.7 & .97 & $.50-1.88$ & .921 & .77 & $.36-1.61$ & .482 \\
\hline & 52 & $\geq 60$ & 2.37 & 32.7 & 23.1 & 19.2 & 25.0 & & & & & & \\
\hline \multirow[t]{2}{*}{ Constipation } & 87 & $<60$ & 2.38 & 17.2 & 40.2 & 29.9 & 12.6 & .67 & $.39-1.14$ & .139 & .74 & $.42-1.32$ & .308 \\
\hline & 96 & $\geq 60$ & 2.19 & 24.0 & 43.8 & 21.9 & 10.4 & & & & & & \\
\hline \multirow[t]{2}{*}{ Swelling of arms or legs } & 42 & $<60$ & 2.19 & 33.3 & 31.0 & 19.0 & 16.7 & .92 & $.43-1.98$ & .837 & .86 & $.36-2.04$ & .734 \\
\hline & 47 & $\geq 60$ & 2.09 & 25.5 & 44.7 & 25.5 & 4.3 & & & & & & \\
\hline \multirow[t]{2}{*}{ I don't look like myself } & 91 & $<60$ & 2.14 & 24.2 & 47.3 & 18.7 & 9.9 & .52 & $.28-.95$ & .033 & .49 & $.25-.96$ & .037 \\
\hline & 62 & $\geq 60$ & 1.82 & 43.5 & 32.3 & 22.6 & 1.6 & & & & & & \\
\hline \multirow[t]{2}{*}{ Changes in skin } & 99 & $<60$ & 2.07 & 24.2 & 53.5 & 13.1 & 9.1 & .63 & $.34-1.16$ & .140 & .80 & $.41-1.56$ & .510 \\
\hline & 61 & $\geq 60$ & 1.89 & 37.7 & 42.6 & 13.1 & 6.6 & & & & & & \\
\hline
\end{tabular}

Abbreviations: $\mathrm{Cl}$, confidence interval; $\mathrm{OR}$, odds ratio.

tSeverity ratings = slight (1), moderate (2), severe (3), very severe (4).

Table 5 Differences in FREQUENCY ratings between younger $(<60, n=263)$ and older $(\geq 60, n=330)$ patients

\begin{tabular}{|c|c|c|c|c|c|c|c|c|c|c|c|c|c|}
\hline \multirow[t]{2}{*}{ Symptom } & \multirow[t]{2}{*}{$\begin{array}{l}\text { Sample } \\
\text { size }\end{array}$} & \multirow[t]{2}{*}{$\begin{array}{l}\text { Age } \\
\text { group }\end{array}$} & \multirow[t]{2}{*}{$\begin{array}{l}\text { Mean } \\
\text { score }\end{array}$} & \multicolumn{4}{|c|}{ Frequency ratingst (\%) } & \multicolumn{3}{|c|}{$\begin{array}{l}\text { Unadjusted values } \\
\text { Older age }\end{array}$} & \multicolumn{3}{|c|}{$\begin{array}{l}\text { Adjusted values } \\
\text { Older age }\end{array}$} \\
\hline & & & & 1 & 2 & 3 & 4 & OR & $\mathrm{Cl}$ & $p$-value & OR & $\mathrm{Cl}$ & $\mathrm{p}$-value \\
\hline \multirow[t]{2}{*}{ Difficulty concentrating } & 150 & $<60$ & 2.19 & 22.7 & 44.0 & 25.3 & 8.0 & .78 & $.50-1.21$ & .269 & .76 & $.46-1.25$ & .277 \\
\hline & 122 & $\geq 60$ & 2.08 & 24.6 & 50.8 & 16.4 & 8.2 & & & & & & \\
\hline \multirow[t]{2}{*}{ Pain } & 192 & $<60$ & 2.64 & 13.0 & 33.3 & 30.2 & 23.4 & .96 & $.67-1.38$ & .829 & .95 & $.63-1.44$ & .825 \\
\hline & 188 & $\geq 60$ & 2.62 & 14.9 & 32.4 & 28.7 & 23.9 & & & & & & \\
\hline \multirow[t]{2}{*}{ Lack of energy } & 215 & $<60$ & 2.82 & 5.1 & 30.2 & 42.3 & 22.3 & .88 & $.62-1.23$ & .446 & .77 & $.53-1.13$ & .179 \\
\hline & 228 & $\geq 60$ & 2.74 & 10.5 & 32.0 & 30.3 & 27.2 & & & & & & \\
\hline \multirow[t]{2}{*}{ Cough } & 106 & $<60$ & 2.12 & 26.4 & 41.5 & 25.5 & 6.6 & .98 & $.61-1.59$ & .937 & .72 & $.42-1.25$ & .246 \\
\hline & 117 & $\geq 60$ & 2.12 & 26.5 & 42.7 & 23.1 & 7.7 & & & & & & \\
\hline \multirow[t]{2}{*}{ Feeling nervous } & 104 & $<60$ & 2.13 & 22.1 & 48.1 & 24.0 & 5.8 & .57 & $.33-.97$ & .038 & .52 & $.27-.99$ & .046 \\
\hline & 88 & $\geq 60$ & 1.92 & 35.2 & 45.5 & 11.4 & 8.0 & & & & & & \\
\hline \multirow[t]{2}{*}{ Dry mouth } & 115 & $<60$ & 2.66 & 13.9 & 26.1 & 40.0 & 20.0 & .66 & $.42-1.03$ & .070 & .52 & $.32-.85$ & .009 \\
\hline & 139 & $\geq 60$ & 2.44 & 20.9 & 31.7 & 30.2 & 17.3 & & & & & & \\
\hline \multirow[t]{2}{*}{ Nausea } & 100 & $<60$ & 2.23 & 23.0 & 40.0 & 28.0 & 9.0 & .55 & $.32-.94$ & .028 & .54 & $.29-.99$ & .047 \\
\hline & 85 & $\geq 60$ & 1.95 & 34.1 & 43.5 & 15.3 & 7.1 & & & & & & \\
\hline \multirow[t]{2}{*}{ Feeling drowsy } & 171 & $<60$ & 2.40 & 11.7 & 44.4 & 36.3 & 7.6 & .82 & $.56-1.22$ & .338 & .65 & $.42-1.01$ & .058 \\
\hline & 176 & $\geq 60$ & 2.34 & 11.9 & 52.3 & 25.6 & 10.2 & & & & & & \\
\hline \multirow[t]{2}{*}{ Numbness/tingling in hands/feet } & 95 & $<60$ & 2.63 & 16.8 & 36.8 & 12.6 & 33.7 & 1.08 & $.65-1.81$ & .771 & .94 & $.52-1.72$ & .846 \\
\hline & 94 & $\geq 60$ & 2.68 & 20.2 & 23.4 & 24.5 & 31.9 & & & & & & \\
\hline \multirow[t]{2}{*}{ Difficulty sleeping } & 168 & $<60$ & 2.70 & 9.5 & 32.1 & 37.5 & 20.8 & .88 & $.59-1.32$ & .541 & .64 & $.41-1.02$ & .058 \\
\hline & 147 & $\geq 60$ & 2.63 & 9.5 & 32.7 & 42.9 & 15.0 & & & & & & \\
\hline Feeling bloated & 84 & $<60$ & 2.39 & 22.6 & 32.1 & 28.6 & 16.7 & .54 & $.29-.98$ & .042 & .49 & $.24-.99$ & .046 \\
\hline
\end{tabular}


Table 5 Differences in FREQUENCY ratings between younger $(<60, n=263)$ and older $(\geq 60, n=330)$ patients (Continued)

\begin{tabular}{|c|c|c|c|c|c|c|c|c|c|c|c|c|c|}
\hline & 63 & $\geq 60$ & 2.06 & 28.6 & 47.6 & 12.7 & 11.1 & & & & & & \\
\hline \multirow[t]{2}{*}{ Problems with urination } & 56 & $<60$ & 2.39 & 21.4 & 33.9 & 28.6 & 16.1 & 1.52 & $.84-2.73$ & .164 & 1.29 & $.68-2.46$ & .438 \\
\hline & 119 & $\geq 60$ & 2.61 & 11.8 & 33.6 & 37.0 & 17.6 & & & & & & \\
\hline \multirow[t]{2}{*}{ Vomiting } & 46 & $<60$ & 1.87 & 39.1 & 41.3 & 13.0 & 6.5 & .85 & $.38-1.93$ & .705 & .46 & $.15-1.38$ & .165 \\
\hline & 36 & $\geq 60$ & 1.81 & 44.4 & 36.1 & 13.9 & 5.6 & & & & & & \\
\hline \multirow[t]{2}{*}{ Shortness of breath } & 99 & $<60$ & 2.17 & 19.2 & 52.5 & 20.2 & 8.1 & 1.30 & $.78-2.14$ & .311 & .97 & $.55-1.70$ & .916 \\
\hline & 106 & $\geq 60$ & 2.35 & 25.5 & 34.0 & 20.8 & 19.8 & & & & & & \\
\hline \multirow[t]{2}{*}{ Diarrhea } & 57 & $<60$ & 2.12 & 22.8 & 47.4 & 24.6 & 5.3 & .56 & $.29-1.10$ & .094 & .47 & $.21-1.03$ & .059 \\
\hline & 72 & $\geq 60$ & 1.89 & 29.2 & 55.6 & 12.5 & 2.8 & & & & & & \\
\hline \multirow[t]{2}{*}{ Feeling sad } & 137 & $<60$ & 2.05 & 28.5 & 42.3 & 24.8 & 4.4 & .99 & $.62-1.59$ & .978 & .79 & $.46-1.36$ & .386 \\
\hline & 103 & $\geq 60$ & 2.08 & 27.2 & 47.6 & 15.5 & 9.7 & & & & & & \\
\hline \multirow[t]{2}{*}{ Sweats } & 119 & $<60$ & 2.39 & 16.8 & 37.0 & 36.1 & 10.1 & .66 & $.41-1.07$ & .093 & .64 & $.37-1.12$ & .121 \\
\hline & 103 & $\geq 60$ & 2.21 & 22.3 & 44.7 & 22.3 & 10.7 & & & & & & \\
\hline \multirow[t]{2}{*}{ Worrying } & 152 & $<60$ & 2.41 & 9.9 & 52.0 & 25.0 & 13.2 & .59 & $.36-.94$ & .028 & .63 & $.37-1.08$ & .094 \\
\hline & 106 & $\geq 60$ & 2.20 & 22.6 & 48.1 & 16.0 & 13.2 & & & & & & \\
\hline \multirow[t]{2}{*}{ Problems with sexual interest } & 93 & $<60$ & 2.86 & 15.1 & 23.7 & 21.5 & 39.8 & 1.07 & $.61-1.86$ & .821 & 1.04 & $.53-2.02$ & .916 \\
\hline & 74 & $\geq 60$ & 2.89 & 17.6 & 17.6 & 23.0 & 41.9 & & & & & & \\
\hline \multirow[t]{2}{*}{ Itching } & 90 & $<60$ & 2.38 & 14.4 & 46.7 & 25.6 & 13.3 & .72 & $.41-1.27$ & .254 & .71 & $.38-1.33$ & .289 \\
\hline & 76 & $\geq 60$ & 2.21 & 23.7 & 40.8 & 26.3 & 9.2 & & & & & & \\
\hline \multirow[t]{2}{*}{ Lack of appetite } & 100 & $<60$ & 2.44 & 18.0 & 39.0 & 24.0 & 19.0 & 1.44 & $.88-2.38$ & .149 & 1.16 & $.66-2.05$ & .598 \\
\hline & 101 & $\geq 60$ & 2.64 & 18.8 & 24.8 & 29.7 & 26.7 & & & & & & \\
\hline \multirow[t]{2}{*}{ Dizziness } & 71 & $<60$ & 1.82 & 39.4 & 40.8 & 18.3 & 1.4 & .96 & $.52-1.78$ & .902 & .78 & $.38-1.61$ & .502 \\
\hline & 70 & $\geq 60$ & 1.81 & 38.6 & 45.7 & 11.4 & 4.3 & & & & & & \\
\hline \multirow[t]{2}{*}{ Difficulty swallowing } & 43 & $<60$ & 2.23 & 30.2 & 32.6 & 20.9 & 16.3 & .87 & $.41-1.81$ & .701 & .56 & $.23-1.38$ & .207 \\
\hline & 50 & $\geq 60$ & 2.14 & 32.0 & 34.0 & 22.0 & 12.0 & & & & & & \\
\hline \multirow[t]{2}{*}{ Feeling irritable } & 136 & $<60$ & 1.99 & 30.1 & 44.1 & 22.1 & 3.7 & .78 & $.48-1.25$ & .300 & .67 & $.38-1.17$ & .161 \\
\hline & 105 & $\geq 60$ & 1.88 & 33.3 & 47.6 & 17.1 & 1.9 & & & & & & \\
\hline \multirow[t]{2}{*}{ Mouth sores } & 39 & $<60$ & 2.21 & 38.5 & 28.2 & 7.7 & 25.6 & .73 & $.33-1.63$ & .447 & .75 & $.29-1.93$ & .553 \\
\hline & 42 & $\geq 60$ & 2.02 & 47.6 & 23.8 & 7.1 & 21.4 & & & & & & \\
\hline \multirow[t]{2}{*}{ Change in way food tastes } & 80 & $<60$ & 2.54 & 17.5 & 31.3 & 31.3 & 20.0 & 1.11 & $.65-1.88$ & .709 & 1.16 & $.65-2.08$ & .616 \\
\hline & 98 & $\geq 60$ & 2.60 & 16.3 & 33.7 & 23.5 & 26.5 & & & & & & \\
\hline \multirow[t]{2}{*}{ Weight loss } & 59 & $<60$ & 2.07 & 22.0 & 55.9 & 15.3 & 6.8 & .76 & $.40-1.44$ & .400 & .56 & $.27-1.16$ & .120 \\
\hline & 75 & $\geq 60$ & 1.99 & 34.7 & 41.3 & 14.7 & 9.3 & & & & & & \\
\hline \multirow[t]{2}{*}{ Hair loss } & 62 & $<60$ & 2.85 & 16.1 & 22.6 & 21.0 & 40.3 & 1.02 & $.53-1.95$ & .953 & .89 & $.43-1.84$ & .745 \\
\hline & 60 & $\geq 60$ & 2.85 & 21.7 & 15.0 & 20.0 & 43.3 & & & & & & \\
\hline \multirow[t]{2}{*}{ Constipation } & 89 & $<60$ & 2.53 & 14.6 & 40.4 & 22.5 & 22.5 & .82 & $.49-1.38$ & .455 & .87 & $.49-1.53$ & .626 \\
\hline & 102 & $\geq 60$ & 2.42 & 15.7 & 45.1 & 20.6 & 18.6 & & & & & & \\
\hline \multirow[t]{2}{*}{ Swelling of arms or legs } & 43 & $<60$ & 2.67 & 23.3 & 23.3 & 16.3 & 37.2 & 1.04 & $.51-2.15$ & .906 & .91 & $.39-2.12$ & .832 \\
\hline & 54 & $\geq 60$ & 2.70 & 24.1 & 20.4 & 16.7 & 38.9 & & & & & & \\
\hline \multirow[t]{2}{*}{ I don't look like myself } & 92 & $<60$ & 2.67 & 15.2 & 29.3 & 28.3 & 27.2 & .79 & $.45-1.39$ & .420 & .84 & $.45-1.57$ & .587 \\
\hline & 69 & $\geq 60$ & 2.54 & 20.3 & 27.5 & 30.4 & 21.7 & & & & & & \\
\hline \multirow[t]{2}{*}{ Changes in skin } & 100 & $<60$ & 2.77 & 15.0 & 24.0 & 30.0 & 31.0 & .69 & $.39-1.20$ & .188 & .97 & $.52-1.82$ & .929 \\
\hline & 67 & $\geq 60$ & 2.54 & 23.9 & 25.4 & 23.9 & 26.9 & & & & & & \\
\hline
\end{tabular}


Table 6 Differences in DISTRESS ratings between younger $(<60, n=263)$ and older $(\geq 60, n=330)$ patients

\begin{tabular}{|c|c|c|c|c|c|c|c|c|c|c|c|c|c|c|}
\hline \multirow[t]{2}{*}{ Symptom } & \multirow[t]{2}{*}{$\begin{array}{l}\text { Sample } \\
\text { size }\end{array}$} & \multirow[t]{2}{*}{$\begin{array}{l}\text { Age } \\
\text { group }\end{array}$} & \multirow[t]{2}{*}{$\begin{array}{l}\text { Mean } \\
\text { score }\end{array}$} & \multicolumn{5}{|c|}{ Distress ratings† (\%) } & \multicolumn{3}{|c|}{$\begin{array}{c}\text { Unadjusted values } \\
\text { Older age }\end{array}$} & \multicolumn{3}{|c|}{$\begin{array}{l}\text { Adjusted values } \\
\text { Older age }\end{array}$} \\
\hline & & & & 0 & 1 & 2 & 3 & 4 & $\overline{O R}$ & $\mathrm{Cl}$ & $\overline{p \text {-value }}$ & OR & $\mathrm{Cl}$ & p-value \\
\hline \multirow[t]{2}{*}{ Difficulty concentrating } & 147 & $<60$ & 1.52 & 17.7 & 38.1 & 22.4 & 18.4 & 3.4 & .71 & $.45-1.10$ & .124 & .82 & $.50-1.34$ & .424 \\
\hline & 116 & $\geq 60$ & 1.33 & 25.0 & 37.9 & 21.6 & 10.3 & 5.2 & & & & & & \\
\hline \multirow[t]{2}{*}{ Pain } & 192 & $<60$ & 2.01 & 6.3 & 32.3 & 29.2 & 19.3 & 13.0 & .63 & $.43-.90$ & .012 & .65 & $.43-.98$ & .041 \\
\hline & 183 & $\geq 60$ & 1.69 & 15.8 & 32.2 & 26.2 & 18.0 & 7.7 & & & & & & \\
\hline \multirow[t]{2}{*}{ Lack of energy } & 212 & $<60$ & 2.04 & 8.5 & 26.4 & 30.2 & 22.6 & 12.3 & .54 & $.39-.77$ & $<.001$ & .57 & $.39-.83$ & .004 \\
\hline & 220 & $\geq 60$ & 1.64 & 20.5 & 30.5 & 22.3 & 18.6 & 8.2 & & & & & & \\
\hline \multirow[t]{2}{*}{ Cough } & 103 & $<60$ & 1.39 & 29.1 & 28.2 & 24.3 & 11.7 & 6.8 & 60 & $.37-.97$ & .037 & .52 & $.29-.91$ & .022 \\
\hline & 112 & $\geq 60$ & 1.07 & 42.9 & 25.0 & 19.6 & 7.1 & 5.4 & & & & & & \\
\hline \multirow[t]{2}{*}{ Feeling nervous } & 102 & $<60$ & 1.70 & 10.8 & 38.2 & 26.5 & 19.6 & 4.9 & .51 & $.30-.87$ & .013 & .57 & $.30-1.06$ & .075 \\
\hline & 85 & $\geq 60$ & 1.33 & 23.5 & 40.0 & 22.4 & 8.2 & 5.9 & & & & & & \\
\hline \multirow[t]{2}{*}{ Dry mouth } & 111 & $<60$ & 1.45 & 22.5 & 37.8 & 20.7 & 9.9 & 9.0 & .50 & $.32-.80$ & .003 & .47 & $.29-.76$ & .002 \\
\hline & 133 & $\geq 60$ & 1.05 & 42.1 & 29.3 & 15.8 & 7.5 & 5.3 & & & & & & \\
\hline \multirow[t]{2}{*}{ Nausea } & 99 & $<60$ & 1.84 & 12.1 & 32.3 & 29.3 & 12.1 & 14.1 & .47 & $.27-80$ & .006 & .46 & $.25-.86$ & .015 \\
\hline & 81 & $\geq 60$ & 1.37 & 33.3 & 29.6 & 13.6 & 13.6 & 9.9 & & & & & & \\
\hline \multirow[t]{2}{*}{ Feeling drowsy } & 165 & $<60$ & 1.39 & 21.2 & 39.4 & 23.0 & 12.1 & 4.2 & .53 & $.36-.79$ & .002 & .52 & $.34-.80$ & .003 \\
\hline & 167 & $\geq 60$ & 1.07 & 40.1 & 31.7 & 13.8 & 9.6 & 4.8 & & & & & & \\
\hline \multirow[t]{2}{*}{ Numbness/tingling in hands/feet } & 94 & $<60$ & 1.40 & 21.3 & 39.4 & 20.2 & 16.0 & 3.2 & .72 & $.43-1.21$ & .212 & .84 & $.46-1.56$ & .586 \\
\hline & 91 & $\geq 60$ & 1.25 & 33.0 & 33.0 & 16.5 & 11.0 & 6.6 & & & & & & \\
\hline \multirow[t]{2}{*}{ Difficulty sleeping } & 166 & $<60$ & 2.01 & 9.6 & 29.5 & 25.9 & 19.9 & 15.1 & .61 & $.41-.92$ & .017 & .54 & $.35-.86$ & .008 \\
\hline & 142 & $\geq 60$ & 1.66 & 19.0 & 27.5 & 29.6 & 16.2 & 7.7 & & & & & & \\
\hline \multirow[t]{2}{*}{ Feeling bloated } & 81 & $<60$ & 1.85 & 16.0 & 24.7 & 27.2 & 22.2 & 9.9 & .43 & $.23-.81$ & .008 & .38 & $.18-.80$ & .011 \\
\hline & 56 & $\geq 60$ & 1.30 & 19.6 & 42.9 & 26.8 & 8.9 & 1.8 & & & & & & \\
\hline \multirow[t]{2}{*}{ Problems with urination } & 54 & $<60$ & 1.57 & 24.1 & 25.9 & 25.9 & 16.7 & 7.4 & 1.21 & $.67-2.19$ & .530 & .91 & $.47-1.76$ & .783 \\
\hline & 108 & $\geq 60$ & 1.70 & 14.8 & 32.4 & 31.5 & 10.2 & 11.1 & & & & & & \\
\hline Vomiting & 43 & $<60$ & 1.63 & 23.3 & 32.6 & 16.3 & 14.0 & 14.0 & .78 & $.34-1.79$ & .553 & .58 & $.21-1.62$ & .298 \\
\hline & 30 & $\geq 60$ & 1.43 & 33.3 & 20.0 & 26.7 & 10.0 & 10.0 & & & & & & \\
\hline Shortness of breath & 97 & $<60$ & 1.63 & 14.4 & 41.2 & 21.6 & 12.4 & 10.3 & .79 & $.48-1.30$ & .347 & .80 & $.46-1.39$ & .427 \\
\hline & 100 & $\geq 60$ & 1.47 & 28.0 & 26.0 & 24.0 & 15.0 & 7.0 & & & & & & \\
\hline Diarrhea & 54 & $<60$ & 1.59 & 18.5 & 38.9 & 14.8 & 20.4 & 7.4 & .69 & $.36-1.34$ & .274 & .61 & $.28-1.31$ & .204 \\
\hline & 63 & $\geq 60$ & 1.32 & 25.4 & 33.3 & 30.2 & 6.3 & 4.8 & & & & & & \\
\hline Feeling sad & 137 & $<60$ & 1.87 & 8.0 & 36.5 & 27.0 & 17.5 & 10.9 & .47 & $.29-.77$ & .003 & .49 & $.28-.85$ & .011 \\
\hline & 94 & $\geq 60$ & 1.41 & 14.9 & 46.8 & 24.5 & 9.6 & 4.3 & & & & & & \\
\hline Sweats & 117 & $<60$ & 1.75 & 17.1 & 29.1 & 28.2 & 12.8 & 12.8 & .49 & $.30-.80$ & .005 & .56 & $.32-.98$ & .040 \\
\hline & 95 & $\geq 60$ & 1.26 & 24.2 & 40.0 & 24.2 & 8.4 & 3.2 & & & & & & \\
\hline Worrying & 151 & $<60$ & 1.93 & 7.3 & 34.4 & 28.5 & 17.9 & 11.9 & .45 & $.28-.72$ & .001 & .57 & $.34-.96$ & .033 \\
\hline & 101 & $\geq 60$ & 1.46 & 8.9 & 53.5 & 23.8 & 10.9 & 3.0 & & & & & & \\
\hline Problems with sexual interest & 91 & $<60$ & 2.08 & 22.0 & 19.8 & 15.4 & 14.3 & 28.6 & .96 & $.55-1.66$ & .875 & .89 & $.46-1.73$ & .731 \\
\hline & 69 & $\geq 60$ & 2.03 & 18.8 & 20.3 & 24.6 & 11.6 & 24.6 & & & & & & \\
\hline Itching & 88 & $<60$ & 1.56 & 15.9 & 40.9 & 25.0 & 8.0 & 10.2 & .82 & $.46-1.45$ & .497 & 1.04 & $.55-1.95$ & .908 \\
\hline & 69 & $\geq 60$ & 1.42 & 24.6 & 31.9 & 27.5 & 8.7 & 7.2 & & & & & & \\
\hline Lack of appetite & 99 & $<60$ & 1.38 & 27.3 & 31.3 & 24.2 & 10.1 & 7.1 & .85 & $.51-1.41$ & .529 & .85 & $.48-1.51$ & .589 \\
\hline & 96 & $\geq 60$ & 1.28 & 32.3 & 29.2 & 21.9 & 11.5 & 5.2 & & & & & & \\
\hline Dizziness & 69 & $<60$ & 1.32 & 23.2 & 37.7 & 26.1 & 10.1 & 2.9 & .84 & $.45-1.56$ & .572 & .62 & $.30-1.29$ & .202 \\
\hline & 63 & $\geq 60$ & 1.25 & 23.8 & 46.0 & 15.9 & 9.5 & 4.8 & & & & & & \\
\hline
\end{tabular}


Table 6 Differences in DISTRESS ratings between younger $(<60, n=263)$ and older $(\geq 60, n=330)$ patients (Continued)

\begin{tabular}{|c|c|c|c|c|c|c|c|c|c|c|c|c|c|c|}
\hline \multirow[t]{2}{*}{ Difficulty swallowing } & 40 & $<60$ & 1.65 & 10.0 & 40.0 & 30.0 & 15.0 & 5.0 & .96 & $.44-2.09$ & .924 & .85 & $.35-2.06$ & .724 \\
\hline & 43 & $\geq 60$ & 1.63 & 20.9 & 27.9 & 23.3 & 23.3 & 4.7 & & & & & & \\
\hline \multirow[t]{2}{*}{ Feeling irritable } & 135 & $<60$ & 1.49 & 21.5 & 34.8 & 24.4 & 11.9 & 7.4 & .79 & $.49-1.27$ & .335 & .82 & $.48-1.42$ & .485 \\
\hline & 96 & $\geq 60$ & 1.31 & 18.8 & 47.9 & 17.7 & 14.6 & 1.0 & & & & & & \\
\hline \multirow[t]{2}{*}{ Mouth sores } & 36 & $<60$ & 1.67 & 22.2 & 30.6 & 19.4 & 13.9 & 13.9 & .46 & $.20-1.09$ & .079 & .41 & $.16-1.03$ & .058 \\
\hline & 35 & $\geq 60$ & 1.11 & 31.4 & 42.9 & 11.4 & 11.4 & 2.9 & & & & & & \\
\hline \multirow[t]{2}{*}{ Change in way food tastes } & 78 & $<60$ & 1.69 & 17.9 & 32.1 & 25.6 & 11.5 & 12.8 & .47 & $.27-.81$ & .007 & .57 & $.31-1.05$ & .072 \\
\hline & 89 & $\geq 60$ & 1.18 & 32.6 & 36.0 & 14.6 & 14.6 & 2.2 & & & & & & \\
\hline \multirow[t]{2}{*}{ Weight loss } & 61 & $<60$ & 1.05 & 45.9 & 26.2 & 13.1 & 6.6 & 8.2 & .67 & $.35-1.29$ & .232 & .51 & $.24-1.09$ & .082 \\
\hline & 68 & $\geq 60$ & 0.78 & 54.4 & 26.5 & 8.8 & 7.4 & 2.9 & & & & & & \\
\hline \multirow[t]{2}{*}{ Hair loss } & 61 & $<60$ & 1.74 & 19.7 & 29.5 & 21.3 & 16.4 & 13.1 & .50 & $.26-.97$ & .041 & .48 & $.23-.99$ & .048 \\
\hline & 55 & $\geq 60$ & 1.29 & 43.6 & 20.0 & 9.1 & 18.2 & 9.1 & & & & & & \\
\hline \multirow[t]{2}{*}{ Constipation } & 87 & $<60$ & 2.09 & 13.8 & 23.0 & 25.3 & 16.1 & 21.8 & .68 & $.41-1.14$ & .145 & .74 & $.42-1.29$ & .285 \\
\hline & 95 & $\geq 60$ & 1.80 & 23.2 & 25.3 & 15.8 & 20.0 & 15.8 & & & & & & \\
\hline \multirow[t]{2}{*}{ Swelling of arms or legs } & 42 & $<60$ & 1.64 & 28.6 & 23.8 & 19.0 & 11.9 & 16.7 & .96 & $.46-2.01$ & .913 & 1.11 & $.46-2.67$ & .819 \\
\hline & 48 & $\geq 60$ & 1.58 & 27.1 & 25.0 & 18.8 & 20.8 & 8.3 & & & & & & \\
\hline \multirow[t]{2}{*}{ I don't look like myself } & 91 & $<60$ & 1.89 & 11.0 & 36.3 & 19.8 & 18.7 & 14.3 & .32 & $.17-.58$ & $<.001$ & .30 & $.15-.58$ & $<.001$ \\
\hline & 64 & $\geq 60$ & 1.14 & 34.4 & 34.4 & 17.2 & 10.9 & 3.1 & & & & & & \\
\hline \multirow[t]{2}{*}{ Changes in skin } & 99 & $<60$ & 1.76 & 11.1 & 36.4 & 29.3 & 12.1 & 11.1 & .41 & $.23-.76$ & .004 & .51 & $.27-.98$ & .043 \\
\hline & 62 & $\geq 60$ & 1.26 & 38.7 & 24.2 & 17.7 & 11.3 & 8.1 & & & & & & \\
\hline
\end{tabular}

Abbreviations: $\mathrm{Cl}$, confidence interval; $\mathrm{OR}$, odds ratio.

†Distress ratings = not at all (0), a little bit (1), somewhat (2), quite a bit (4), very much (5).

dimensions of the symptom experience in a very large sample of oncology patients, several limitations need to be acknowledged before our findings are placed within the context of an extremely limited literature. First, detailed information was not available on the exact number or specific types of comorbidities these patients experienced. In addition, specific details on the doses of CTX and RT received by these patients were not collected for these studies. Despite these limitations, findings from this initial analysis can be used to generate testable hypotheses for future research.

For almost $50 \%$ of the MSAS symptoms, older patients reported significantly lower occurrence rates. However, eight of the eleven symptoms with the highest occurrence rates were identical in the two age groups (i.e., lack of energy, pain, feeling drowsy, difficulty sleeping, dry mouth, difficulty concentrating, worrying, feeling irritable). Consistent with previous studies of multiple symptoms [15,35-39], these eight symptoms are among the most common symptoms reported by oncology patients regardless of cancer diagnosis, stage of disease, and/or cancer treatment. Since these eight symptom occurrence rates exhibit a similar distribution pattern in both age groups, one plausible hypothesis for the significantly lower occurrence rates for six of these symptoms in older patients (no difference in lack of energy or dry mouth) is that older patients received lower doses of CTX or RT $[3,12,14,40]$. An equally plausible hypothesis is that older patients received comparable doses of therapy. However, because of age-related changes in a number of biological processes [41] and/or a variety of psychosocial factors [42,43], older patients reported lower symptom occurrence rates. For example, agerelated changes may occur in the hypothalamic-adrenal-

Table 7 Differences in subscale and total scores on the Memorial Symptom Assessment Scale (MSAS) between younger $(<60, n=263)$ and older $(\geq 60, n=330)$ patients

\begin{tabular}{|c|c|c|c|c|c|c|}
\hline \multirow[t]{3}{*}{ Scale } & \multicolumn{3}{|c|}{ Unadjusted scores } & \multicolumn{3}{|c|}{ Adjusted scores } \\
\hline & \multicolumn{3}{|c|}{ Mean (Standard deviation) } & \multicolumn{3}{|c|}{ Mean (Standard error) } \\
\hline & $<60$ years & $\geq 60$ years & p-value & $<60$ years & $\geq 60$ years & p-value \\
\hline Physical subscale & $.92(.63)$ & $.70(.58)$ & $<.0001$ & $.90(.04)$ & $.70(.03)$ & $<.0001$ \\
\hline Psychological subscale & $1.15(.84)$ & $.67(.69)$ & $<.0001$ & $1.10(.05)$ & $.70(.04)$ & $<.0001$ \\
\hline Global Distress Index & $1.17(.74)$ & $.78(.70)$ & $<.0001$ & $1.13(.04)$ & $.81(.04)$ & $<.0001$ \\
\hline Total MSAS score & $.84(.50)$ & $.60(.46)$ & $<.0001$ & $.81(.03)$ & $.60(.03)$ & $<.0001$ \\
\hline
\end{tabular}


Table 8 Differences between younger $(<60, n=263)$ and older ( $\geq 60, n=330$ ) patients in rankings of symptoms with the highest occurrence, severity, frequency, and distress ratings

\begin{tabular}{|c|c|c|c|c|}
\hline \multicolumn{5}{|c|}{ OCCURRENCE RATINGS } \\
\hline \multirow[t]{2}{*}{ Rank } & \multicolumn{2}{|l|}{$<60$} & \multicolumn{2}{|l|}{$\geq 60$} \\
\hline & Symptom & $\begin{array}{l}\% \text { of } \\
\text { patients }\end{array}$ & Symptom & $\begin{array}{l}\% \text { of } \\
\text { patients }\end{array}$ \\
\hline 1 & Lack of energy & 81.7 & Lack of energy & 69.1 \\
\hline 2 & Pain & 73.0 & Pain & 57.0 \\
\hline 3 & Feeling drowsy & 65.0 & Feeling drowsy & 53.6 \\
\hline 4 & Difficulty sleeping & 63.9 & Difficulty sleeping & 44.8 \\
\hline 5 & Worrying & 57.8 & Dry mouth & 42.1 \\
\hline 6 & $\begin{array}{c}\text { Difficulty } \\
\text { concentrating }\end{array}$ & 57.0 & $\begin{array}{c}\text { Difficulty } \\
\text { concentrating }\end{array}$ & 37.0 \\
\hline 7 & Feeling sad & 52.1 & $\begin{array}{c}\text { Problems with } \\
\text { urination }\end{array}$ & 36.1 \\
\hline 8 & Feeling irritable & 51.7 & Cough & 35.5 \\
\hline 9 & Sweats & 45.2 & Shortness of breath & 32.1 \\
\hline \multirow[t]{2}{*}{10} & Dry mouth & 43.7 & Worrying & 32.1 \\
\hline & & & Feeling irritable & 32.1 \\
\hline \multicolumn{5}{|c|}{ SEVERITY RATINGS } \\
\hline Rank & Symptom & $\begin{array}{l}\text { Mean } \\
\text { score }\end{array}$ & Symptom & $\begin{array}{l}\text { Mean } \\
\text { score }\end{array}$ \\
\hline 1 & $\begin{array}{c}\text { Problems with sexual } \\
\text { interest }\end{array}$ & 2.50 & $\begin{array}{c}\text { Problems with sexual } \\
\text { interest }\end{array}$ & 2.72 \\
\hline 2 & Hair loss & 2.38 & Hair loss & 2.37 \\
\hline 3 & Constipation & 2.38 & Constipation & 2.19 \\
\hline 4 & Difficulty sleeping & 2.23 & Difficulty sleeping & 2.16 \\
\hline 5 & $\begin{array}{l}\text { Swelling of arms or } \\
\text { legs }\end{array}$ & 2.19 & Lack of energy & 2.13 \\
\hline 6 & Lack of energy & 2.15 & Pain & 2.12 \\
\hline 7 & Sweats & 2.14 & $\begin{array}{l}\text { Swelling of arms or } \\
\text { legs }\end{array}$ & 2.09 \\
\hline 8 & $\begin{array}{l}\text { I don't look like } \\
\text { myself }\end{array}$ & 2.14 & $\begin{array}{c}\text { Problems with } \\
\text { urination }\end{array}$ & 2.01 \\
\hline 9 & Worrying & 2.10 & Shortness of breath & 2.00 \\
\hline 10 & Pain & 2.08 & Lack of appetite & 2.00 \\
\hline \multicolumn{5}{|c|}{ FREQUENCY RATINGS } \\
\hline Rank & Symptom & $\begin{array}{l}\text { Mean } \\
\text { score }\end{array}$ & Symptom & $\begin{array}{l}\text { Mean } \\
\text { score }\end{array}$ \\
\hline 1 & $\begin{array}{c}\text { Problems with sexual } \\
\text { interest }\end{array}$ & 2.86 & $\begin{array}{c}\text { Problems with sexual } \\
\text { interest }\end{array}$ & 2.89 \\
\hline 2 & Hair loss & 2.85 & Hair loss & 2.85 \\
\hline 3 & Lack of energy & 2.82 & Lack of energy & 2.74 \\
\hline 4 & Changes in skin & 2.77 & $\begin{array}{l}\text { Swelling of arms or } \\
\text { legs }\end{array}$ & 2.70 \\
\hline 5 & Difficulty sleeping & 2.70 & $\begin{array}{l}\text { Numbness/tingling } \\
\text { in hands/feet }\end{array}$ & 2.68 \\
\hline 6 & $\begin{array}{l}\text { Swelling of arms or } \\
\text { legs }\end{array}$ & 2.67 & Lack of appetite & 2.64 \\
\hline 7 & $\begin{array}{l}\text { I don't look like } \\
\text { myself }\end{array}$ & 2.67 & Difficulty sleeping & 2.63 \\
\hline 8 & Dry mouth & 2.66 & Pain & 2.62 \\
\hline
\end{tabular}

Table 8 Differences between younger $(<60, n=263)$ and older $(\geq 60, n=330)$ patients in rankings of symptoms with the highest occurrence, severity, frequency, and distress ratings (Continued)

\begin{tabular}{|c|c|c|c|c|}
\hline 9 & Pain & 2.64 & $\begin{array}{c}\text { Problems with } \\
\text { urination }\end{array}$ & 2.61 \\
\hline 10 & $\begin{array}{c}\text { Numbness/tingling in } \\
\text { hands/feet }\end{array}$ & 2.63 & Changes in taste & 2.60 \\
\hline \multicolumn{5}{|c|}{ DISTRESS RATINGS } \\
\hline Rank & Symptom & $\begin{array}{l}\text { Mean } \\
\text { score }\end{array}$ & Symptom & $\begin{array}{l}\text { Mean } \\
\text { score }\end{array}$ \\
\hline 1 & Constipation & 2.09 & $\begin{array}{c}\text { Problems with sexual } \\
\text { interest }\end{array}$ & 2.03 \\
\hline 2 & $\begin{array}{l}\text { Problems with sexual } \\
\text { interest }\end{array}$ & 2.08 & Constipation & 1.80 \\
\hline 3 & Lack of energy & 2.04 & $\begin{array}{c}\text { Problems with } \\
\text { urination }\end{array}$ & 1.70 \\
\hline 4 & Pain & 2.01 & Pain & 1.69 \\
\hline 5 & Difficulty sleeping & 2.01 & Difficulty sleeping & 1.66 \\
\hline 6 & Worrying & 1.93 & Lack of energy & 1.64 \\
\hline 7 & $\begin{array}{c}\text { I don't look like } \\
\text { myself }\end{array}$ & 1.89 & Difficulty swallowing & 1.63 \\
\hline 8 & Feeling sad & 1.87 & $\begin{array}{l}\text { Swelling of arms or } \\
\text { legs }\end{array}$ & 1.58 \\
\hline 9 & Feeling bloated & 1.85 & Shortness of breath & 1.47 \\
\hline 10 & Nausea & 1.84 & Worrying & 1.46 \\
\hline
\end{tabular}

pituitary axis (HPA) that mediate the occurrence and severity of the most common cancer-related symptoms [44].

Another plausible hypothesis for the lower symptom occurrence rates in older patients is that older persons may experience a "response shift" in their perception of symptoms. A "response shift" is defined as an agerelated psychological shift that represents a change in a person's internal framework for the assessment of experiences [45]. This concept was first used in oncology to describe changes over time in QOL [46]. In the context of older patients' reports of symptoms, these individuals may have experienced an internal reconceptualization of their symptom experience based on their lifetime experience with symptoms or their experience with symptoms from other chronic medical conditions [45]. In addition, based on studies of barriers to pain assessment and management in older adults [47], older patients tend to under-report pain because: they view it as a normal part of aging; they are concerned that if they report pain it will distract their clinician from treating their cancer; they are fearful about additional diagnostic tests and their associated costs; or they are worried about additional treatments (e.g., opioid analgesics for pain) and associated adverse events $[11,48,49]$. It is plausible that these same barriers contribute to under-reporting of other symptoms in older adults. Cough and shortness of breath were among the top most 
frequently occurring symptoms only in the older age group. In contrast, feeling sad and sweats were unique to the younger age group. These differences in patterns of occurrence warrant investigation in future studies.

For older and younger patients who reported a specific symptom, severity ratings for the majority of the symptoms were mild to moderate. For all but six symptoms (i.e., dry mouth, feeling drowsy, feeling sad, sweats, worrying, I don't look like myself), no age-related differences in severity scores were reported (see Table 3). In addition to the hypotheses stated above, several potential explanations for the lack of differences in symptom severity ratings warrant consideration. If younger patients received higher doses of $\mathrm{RT}$ and/or CTX than older patients, one would expect higher symptom severity scores in the younger group. One potential explanation for the equivalent symptom severity scores is that younger patients received more aggressive symptom management than older patients. This hypothesis is supported by studies that found that older patients receive lower doses of opioid analgesics than younger patients with comparable pain severity scores [50,51].

In terms of the occurrence rates for specific symptoms (see Table 8), seven of the ten symptoms with the highest severity ratings were the same in both age groups (i.e., problems with sexual interest, hair loss, constipation, difficulty sleeping, swelling of arms or legs, lack of energy, pain). A similar pattern for the most severe symptoms across age groups suggests that these symptoms are common across cancer treatments. The age differential in terms of actual severity ratings may be related to dose reductions in the elderly or due to other biological or psychological mechanisms described above. Similar hypotheses could be proposed to explain the age-related differences in the symptom frequency ratings.

While a "response shift" in older persons' perceptions of their symptoms is a plausible explanation for the lower occurrence rates for over $50 \%$ of the MSAS symptoms, one might ask why this same hypothesis does not seem to apply for the other dimensions of the symptom experience. One reason that a "response shift" may not be as evident in patients' ratings of symptom severity, frequency, and distress is that the age group comparisons for these three dimensions were done with only patients who reported the symptom. To verify this hypothesis, differences in symptom severity scores between older and younger patients were re-analyzed with the inclusion of patients who did not report each symptom (i.e., symptom severity scores ranged from 0 to 4 instead of from 1 to 4). In these analyses, compared to younger patients, older patients reported significantly lower severity scores for: difficulty concentrating, pain, lack of energy, feeling nervous, nausea, feeling drowsy, difficulty sleeping, feeling bloated, vomiting, feeling sad, problems with sexual interest, feeling irritable, worrying, I don't look like myself, and changes in skin. This finding suggests that in older patients who develop a symptom as a result of their cancer or its treatment, they experience it with a similar severity as younger patients. The reasons why some older patients do and do not experience cancer symptoms warrants investigation in future studies.

Besides occurrence, the largest number of age-related differences were found in the symptom distress ratings (i.e., for $43.8 \%$ of the symptoms, older patients reported significantly lower ratings). Symptom distress is defined as the degree or amount of physical or mental upset, anguish, or suffering experienced from a specific symptom $[26,52]$. In one review [39], out of 22 studies that assessed multiple symptoms in cancer patients, only seven (31.8\%) assessed distress. Of those seven, three used the MSAS [39]. While no studies were found that evaluated for age differences in MSAS symptom distress ratings, the symptoms with the highest distress ratings in our study are comparable to those found in other studies that used the MSAS [53-55].

As shown in Table 8, six of the ten most distressing symptoms were the same in both age groups (i.e., constipation, problems with sexual interest, lack of energy, pain, difficulty sleeping, worrying). Problems with urination, difficulty swallowing, swelling of the arms or legs, and shortness of breath were among the top most distressing symptoms in the older age group. In contrast, I don't look like myself, feeling sad, feeling bloated, and nausea were unique to the younger age group. These differences in distress patterns warrant additional research.

Of note, no age-related differences were found in symptom distress ratings for constipation and problems with sexual interest. These two symptoms which occurred in approximately $30 \%$ of the patients in both age groups warrant a careful assessment because when they do occur, they are severe, occur frequently, and are distressing not only to older patients, but to younger patients as well.

A surprising finding in this study was that a significantly higher percentage (26\%) of older patients reported being fully active compared to younger patients (15.8\%). While older individuals in the general population are more likely to report poorer functional status than younger individuals [56], the higher functional status in the older patients in this study may be attributed to lower recruitment rates among the oldest old. An alternative explanation is that younger patients received higher doses of CTX or RT that had a negative impact on their functional status.

Despite the limitations enumerated earlier in the discussion, findings from this study provide valuable information to guide clinical practice and research. As shown in Table 8, across all four symptom dimensions, the 
most common, severe, frequent, and distressing symptoms are similar for both age groups. What differs is the magnitude estimation for each dimension, with older persons reporting lower rates of occurrence, severity, frequency, and distress for some symptoms. Because the age-related differences in symptom severity, frequency, and distress scores were small, additional research is warranted within each age group to determine the impact of each dimension of a symptom on younger and older patients' functional status and QOL.

The findings summarized in Table 8 provide new information on symptoms with the highest occurrence, severity, frequency and distress ratings in both age groups. This information can be used by clinicians to guide their multidimensional symptom assessments of older oncology patients. Future research needs to focus on a detailed evaluation of patient (i.e., phenotypic and genotypic characteristics) and clinical characteristics that explain the age differences in the various dimensions of the symptom experience described in this study.

\section{Conclusions}

This study is the first to evaluate for differences in multiple dimensions of symptom experience in older oncology patients. For almost $50 \%$ of the MSAS symptoms, older patients reported significantly lower occurrence rates. While fewer age-related differences were found in ratings of symptom severity, frequency, and distress, a similar pattern was found across all three dimensions. Future research needs to focus on a detailed evaluation of patient and clinical characteristics (i.e., type and dose of treatment) that explain the differences in symptom experience identified in this study.

\section{Abbreviations}

CTX: Chemotherapy; ECOG: Eastern Cooperative Oncology Group: FPS: Fatigue, Pain, and Sleep; HPA: Hypothalamic-adrenal-pituitary axis; MSAS: Memorial Symptom Assessment Scale; QOL: Quality of life; RT: Radiation therapy; UCSF: University of California, San Francisco.

\section{Competing interests}

The authors declare that there are no competing interests.

\section{Authors' contributions}

$\mathrm{JC}$ drafted the manuscript, and participated in the interpretation of the data. SP combined the datasets, conducted data analyses, and contributed to the interpretation of the data, and the critical revision of the manuscript. BC conducted analyses and participated in the critical revision of the manuscript. HS contributed to the analysis, the interpretation of the data, and critical revision of the manuscript. KA, BA, VB, JM, and LD contributed to the interpretation of the data, and the critical revision of the manuscript, and CR participated in the critical revision of the manuscript. PY and CM conceived the concept and study design, coordinated the study, and contributed to the interpretation, drafting, and critical revision of the manuscript. All authors read and approved the final manuscript.

\section{Acknowledgements}

This collaborative project was funded by Atlantic Philanthropies, and a Queensland University of Technology Institute of Health and Biomedical Innovation Human Health and Wellbeing Collaborative Grant Scheme 2010. The Australian study was funded under a Palliative Care National Health and
Medical Research Council grant. The FPS study was funded by the National Institute of Nursing Research (NR04835).

\section{Author details}

${ }^{1}$ School of Nursing, University of California, 2 Koret Way - N631Y, San Francisco, CA 94143-0610, USA. ${ }^{2}$ Schools of Nursing Medicine, University of California, 2 Koret Way - N631Y, San Francisco, CA 94143-0610, USA. ${ }^{3}$ School of Nursing, Queensland University of Technology, Victoria Park Road, Kelvin Grove 4059Queensland, Australia. ${ }^{4}$ Institute for Human Genetics, University of California, 2 Koret Way - N631Y, San Francisco, CA 94143-0610, USA. ${ }^{5}$ Department of Physiological Nursing, University of California, 2 Koret Way - N631Y, San Francisco, CA 94143-0610, USA.

Received: 20 December 2012 Accepted: 20 December 2012

Published: 3 January 2013

\section{References}

1. Jemal A, Bray F, Center MM, Ferlay J, Ward E, Forman D: Global cancer statistics. CA Cancer J Clin 2011, 61:69-90.

2. Balducci L: Supportive care in elderly cancer patients. Curr Opin Oncol 2009, 21:310-317.

3. Townsley C, Pond G, Peloza B, Kok J, Naidoo K, Dale D, Herbert C, Holowaty E, Straus S, Siu L: Analysis of treatment practices for elderly cancer patients in Ontario, Canada. J Clin Oncol 2005, 23:3802-3810.

4. Townsley G, Beck S, Watkins J: "Learning to live with it": coping with the transition to cancer survivorship in older adults. J Aging Stud 2007, 21:93-106.

5. Langer CJ: Clinical evidence on the undertreatment of older and poor performance patients who have advanced non-small-cell lung cancer: Is there a role for targeted therapy in these cohorts? Clin Lung Cancer 2011, 12:272-279.

6. Dale DC: Poor prognosis in elderly patients with cancer: the role of bias and undertreatment. J Support Oncol 2003, 1(suppl 2):11-17.

7. Bastiaannet E, Portielje JEA, van de Velde CJH, de Craen AJM, van der Velde S, Kuppen PJK, van der Geest LGM, Janssen-Heijnen MLG, Dekkers OM, Westendorp RGJ: Lack of survival gain for elderly women with breast cancer. Oncologist 2011, 16:415-423.

8. Kagan SH: Ageism in cancer care. Sem Oncol Nurs 2008, 24:246-253.

9. Kroenke CH, Rosner B, Chen WY, Kawachi I, Colditz GA, Holmes MD: Functional impact of breast cancer by age at diagnosis. J Clin Oncol 2004, 22:1849-1856.

10. Cheung WY, Le LW, Gagliese L, Zimmermann C: Age and gender differences in symptom intensity and symptom clusters among patients with metastatic cancer. Support Care Cancer 2011, 19:417-423.

11. Carreca I, Balducci L: Cancer chemotherapy in the older cancer patient. Urol Oncol 2009, 27:633-642.

12. Brunello A, Loaldi E, Balducci L: Dose adjustment and supportive care before and during treatment. Cancer Treat Rev 2009, 35:493-498.

13. Kumar A, Soares H, Balducci L, Djulbegovic B: Treatment tolerance and efficacy in geriatric oncology: a systematic review of phase III randomized trials conducted by five National Cancer Institute-sponsored cooperative groups. J Clin Oncol 2007, 25:1272-1276.

14. Rose J, O'Toole E, Dawson N, Thomas C, Connors A Jr, Wenger N, Phillips R, Hamel M, Cohen H, Lynn J: Age differences in care practices and outcomes for hospitalized patients with cancer. J Am Geriatr Soc 2000, 48:S25-S32.

15. Mohile SG, Heckler C, Fan L, Mustian K, Jean-Pierre P, Usuki K, Sprod L, Janelsins M, Purnell J, Peppone $L$, et al: Age-related differences in symptoms and their interference with quality of life in 903 cancer patients undergoing radiation therapy. J Geriatr Oncol 2011, 2:225-232.

16. Degner L, Sloan J: Symptom distress in newly diagnosed ambulatory cancer patients and as a predictor of survival in lung cancer. $J$ Pain Symptom Manage 1995, 10:423-431.

17. Yan H, Sellick K: Symptoms, psychological distress, social support, and quality of life of Chinese patients newly diagnosed with gastrointestinal cancer. Cancer Nurs 2004, 27:389-399.

18. Avis NE, Crawford S, Manuel J: Quality of life among younger women with breast cancer. J Clin Oncol 2005, 23:3322-3330.

19. Ellis J, Lin J, Walsh A, Lo C, Shepherd FA, Moore M, Li M, Gagliese L, Zimmermann C, Rodin G: Predictors of referral for specialized psychosocial oncology care in patients with metastatic cancer: the 
contributions of age, distress, and marital status. J Clin Oncol 2009, 27:699-705.

20. Graves KD, Arnold SM, Love CL, Kirsh KL, Moore PG, Passik SD: Distress screening in a multidisciplinary lung cancer clinic: prevalence and predictors of clinically significant distress. Lung Cancer 2007, 55:215-224.

21. Politi MC, Enright TM, Weihs KL: The effects of age and emotional acceptance on distress among breast cancer patients. Support Care Cancer 2007, 15:73-79.

22. Goldzweig G, Hubert A, Walach N, Brenner B, Perry S, Andritsch E, Baider L: Gender and psychological distress among middle-and older-aged colorectal cancer patients and their spouses: an unexpected outcome. Crit Rev Oncol Hematol 2009, 70:71-82.

23. Weiss T, Weinberger MI, Holland J, Nelson C, Moadel A: Falling through the cracks: a review of psychological distress and psychosocial service needs in older Black and Hispanic patients with cancer. J Geriatr Oncol 2012, 3(2):163-173.

24. Yabroff KR, Lamont EB, Mariotto A, Warren JL, Topor M, Meekins A, Brown $\mathrm{ML}$ : Cost of care for elderly cancer patients in the United States. J Nat Cancer Inst 2008, 100:630-641.

25. Smith BD, Smith GL, Hurria A, Hortobagyi GN, Buchholz TA: Future of cancer incidence in the United States: burdens upon an aging, changing nation. J Clin Oncol 2009, 27:2758-2765.

26. Portenoy RK, Thaler HT, Kornblith AB, McCarthy Lepore J, Friedlander-Klar H, Kiyasu E, Sobel K, Coyle N, Kemeny N, Norton L: The memorial symptom assessment scale: an instrument for the evaluation of symptom prevalence, characteristics and distress. Eur J Cancer 1994, 30:1326-1336.

27. Kim H, Barsevick A, Tulman L: Predictors of the intensity of symptoms in a cluster in patients with breast cancer. J Nurs Scholar 2009, 41:158-165.

28. Brant J, Beck S, Dudley W, Cobb P, Pepper G, Miaskowski C: Symptom trajectories in posttreatment cancer survivors. Cancer Nurs 2010, 34:67-77.

29. Hopwood P, Haviland J, Mills J, Sumo G: The impact of age and clinical factors on quality of life in early breast cancer: an analysis of 2208 women recruited to the UK START Trial (Standardisation of Breast Radiotherapy Trial). Breast 2007, 16:241-251.

30. Gagliese $L$, Jovellanos M, Zimmermann C, Shobbrook C, Warr D, Rodin G: Age-related patterns in adaptation to cancer pain: a mixed method study. Pain Med 2009, 10:1050-1061.

31. Gagliese L, Melzack R: Age-related differences in the qualities but not the intensity of chronic pain. Pain 2003, 104:597-608.

32. Zubrod CG, Schneiderman M, Frei E, Brindley C, Gold GL, Shnider B: Appraisal of methods for the study of chemotherapy of cancer in man: comparative therapeutic trial of nitrogen mustard and triethylene thiophosphoramide. J Chron Dis 1960, 11:7-33.

33. Verger E, Salamero M, Conill C: Can Karnofsky performance status be transformed to the Eastern Cooperative Oncology Group scoring scale and vice versa? Eur J Cancer 1992, 28:1328-1330.

34. Hamilton W, Lancashire R, Sharp D, Peters TJ, Cheng K, Marshall T: The risk of colorectal cancer with symptoms at different ages and between the sexes: a case-control study. BMC Med 2009, 7(1):17.

35. Stark LL, Tofthagen C, Visovsky C, McMillan SC: The symptom experience of patients with cancer. J Hosp Palliat Nurs 2012, 14:61-70.

36. Dodd MJ, Miaskowski C, Paul SM: Symptom clusters and their effect on the functional status of patients with cancer. Oncol Nurs Forum 2001, 28:465-470

37. Dodd MJ, Miaskowski C, Lee KA: Occurrence of symptom clusters. JNCI Monographs 2004, 32:76-78.

38. Kim EJ, Dodd M, Aouizerat B, Jahan T, Miaskowski C: A review of the prevalence and impact of multiple symptoms in oncology patients. J Pain Symptom Manage 2009, 37:715-736.

39. Gilbertson-White $S$, Aouizerat $B E$, Jahan T, Miaskowski $C$ : A review of the literature on multiple symptoms, their predictors, and associated outcomes in patients with advanced cancer. Palliat Support Care 2011, 9:81-102.

40. Hamaker M, Schreurs W, van Slooten H, Uppelschoten J, Smorenburg C: Trends in breast cancer treatment in the elderly at a breast cancer outpatient clinic: guidelines followed better. Ned Tijdsch Geneeskd 2009, 153:A562.

41. MCLachlan AJ, Pont LG: Drug metabolism in older people-a key consideration in achieving optimal outcomes with medicines. J Gerontol A Biol Sci Med Sci 2012, 67:175-180.
42. Kahana E, Kahana B, Kelley-Moore J, Adams SA, Hammel R, Kulle D, Brown JA, King C: Toward advocacy in cancer care for older adults: survivors have cautious personal actions but bold advice for others. J Am Geriatr Soc 2009, 57:S269-S271.

43. Lenze EJ, Wetherell $\mathrm{J}$ : A lifespan view of anxiety disorders. Dialogues Clin Neurosci 2011, 13:381-399.

44. Bower JE, Low CA, Moskowitz JT, Sepah S, Epel E: Benefit finding and physical health: positive psychological changes and enhanced allostasis. Soc Personal Psychol Compass 2008, 2:223-244.

45. Costanzo ES, Ryff CD, Singer BH: Psychosocial adjustment among cancer survivors: findings from a national survey of health and well-being. Health Psycho 2009, 28:147-156.

46. Schwartz CE, Sprangers MAG: Methodological approaches for assessing response shift in longitudinal health-related quality-of-life research. Soc Sci Med 1999, 48:1531-1548.

47. Herr K, Titler MG, Schilling ML, Marsh JL, Xie X, Ardery G, Clarke WR, Everett LQ: Evidence-based assessment of acute pain in older adults: current nursing practices and perceived barriers. Clin J Pain 2004, 20:331-340.

48. Pain(PDQ). http://www.cancer.gov/cancertopics/pdq/supportivecare/pain/ HealthProfessional/page1.

49. Ferrell BA: Pain management in elderly people. J Am Geriatr Soc 1991, 39:64-73.

50. Urban D, Cherny N, Catane R: The management of cancer pain in the elderly. Crit Rev Oncol Hematol 2010, 73:176-183.

51. Delgado-Guay MO, Bruera E: Management of pain in the older person with cancer. Part 1. Oncology 2008, 22:148-152.

52. Rhodes VA, Watson PM: Symptom distress-the concept: past and present. Sem Oncol Nurs 1987, 3:242-247.

53. McMillan SC, Small BJ: Symptom distress and quality of life in patients with cancer newly admitted to hospice home care. Oncol Nurs Forum 2002, 29:1421-1428.

54. Peters $L$, Sellick K: Quality of life of cancer patients receiving inpatient and home-based palliative care. J Adv Nurs 2006, 53:524-533.

55. Rodin G, Zimmermann C, Rydall A, Jones J, Shepherd FA, Moore M, Fruh M, Donner A, Gagliese L: The desire for hastened death in patients with metastatic cancer. J Pain Symptom Manage 2007, 33:661-675.

56. Sergi G, Sarti S, Mosele M, Ruggiero E, Imoscopi A, Miotto F, Bolzetta F, Inelmen EM, Manzato E, Coin A: Changes in healthy elderly women's physical performance: a 3-year follow-up. Exp Gerontol 2011, 46:929-933.

doi:10.1186/1471-2407-13-6

Cite this article as: Cataldo et al:: Differences in the symptom experience of older versus younger oncology outpatients: a crosssectional study. BMC Cancer 2013 13:6.

\section{Submit your next manuscript to BioMed Central and take full advantage of:}

- Convenient online submission

- Thorough peer review

- No space constraints or color figure charges

- Immediate publication on acceptance

- Inclusion in PubMed, CAS, Scopus and Google Scholar

- Research which is freely available for redistribution 